\title{
A Theoretical Evaluation of the Efficiencies of Metal- Free 1,3,4-Oxadiazole Dye-Sensitized Solar Cells: Insights From Electron-Hole Separation Distance Analysis.
}

\section{Louis-Charl Coetzee ( $\sim$ Louischarlc0@gmail.com )}

University of Johannesburg https://orcid.org/0000-0003-3003-3025

Adedapo Adeyinka

University of Johannesburg

Nomampondo Magwa

University of South Africa

\section{Research Article}

Keywords: DFT, TD-DFT, DSSCs, Donor, r-spacer, acceptor

Posted Date: December 17th, 2021

DOI: https://doi.org/10.21203/rs.3.rs-1137349/v1

License: (c) (1) This work is licensed under a Creative Commons Attribution 4.0 International License.

Read Full License 


\title{
A theoretical evaluation of the efficiencies of metal-free 1,3,4-oxadiazole dye- sensitized solar cells: insights from electron-hole separation distance analysis.
}

\author{
Louis-Charl Coetzee ${ }^{\mathrm{a}}$, Adedapo Adeyinka ${ }^{\mathrm{a}}$, Nomampondo Magwa ${ }^{\mathrm{b}}$, \\ ${ }^{a}$ Research Centre for Synthesis and Catalysis, Department of Chemical Sciences, University of \\ Johannesburg, PO Box 524, Auckland Park, Johannesburg, 2006, Gauteng, South Africa.
}

Tel.: +27604699790

${ }^{b}$ Department of Chemistry, University of South Africa, Private Bag X6, Florida, Roodepoort, Johannesburg, 1710, Gauteng, South Africa.

Tel.: $+27(011) 6709302$

Louis-Charl Coetzee

Louischarlc0@gmail.com

\begin{abstract}
Herein, some novel metal-free 1,3,4-oxadiazole compounds 01-07 were evaluated for Photovoltaic properties using density functional theory (DFT) and time-dependent density functional theory (TD-DFT) calculations to determine if they can serve as metal-free organic dyes for the use of dye-sensitized solar cells (DSSCs). To understand the trends in the relative efficiencies of the investigated compounds as dyes in DSSCs, their electron contributions, hole contributions, and electron-hole overlaps for each respective atom and fragment within the molecule were analyzed with a particular focus on the electron densities on the anchoring segments. As transition density matrices (TDM) provide details for the departure of each electron from its corresponding hole during excitations, which results in charge transfer (CT), the charge separation distance $(\Delta r)$ between the electron and its corresponding hole was studied as well as the degree of electron-hole overlap $(\Lambda)$. The latter, single-point excitation energy of each electron, the percentage electron contribution to the anchoring segments of each compound, the incident-photon-conversionefficiency $(I P C E)$, charge recombination, light harvesting efficiency $(L H E)$ electron injection $\left(\Phi_{i n j}\right)$ and charge collection efficiency $\left(n_{\text {collect }}\right.$ ) were then compared to $\Delta r$ to determine whether the expected relationships hold. Moreover, parameters such as diffusion constant $\left(D_{\pi}\right)$ and electron lifetime $(t)$, amongst others, were also used to describe electron excitation processes. Since IPCE is the key parameter in determining the efficiency, $\mathbf{O 3}$ was found to be the best dye due to its highest value.
\end{abstract}

Keywords DFT, TD-DFT, DSSCs, Donor, $\pi$-spacer, acceptor

\section{Introduction}

Despite fossil fuels still largely being the most widely used energy source worldwide, there has been concern about whether reserves will soon run out, given that they are limited [1]. Additionally, widespread concerns have also been raised about the pervasiveness of global warming caused by its energy consumption [2]. Unlike fossil fuels, renewable energy sources have several advantages: 
they replenish themselves naturally, produce little secondary waste, and reduce greenhouse gas emissions and promote sustainability $[3,4]$. These include bioenergy, direct solar energy, geothermal energy, hydropower, wind, and ocean energy (tide and wave) [4]. Photovoltaic (PV) systems and solar collectors are two direct solar energy sources. Both systems generate power through the photoelectric effect. The first-generation PV systems contained silicon and are still mostly used due to their hole transporting and charge carrier mobility [5]. However, the challenge in fabricating silicon solar cells and their high fabrication cost makes it difficult to distribute PV systems that contain silicon. In response to these challenges, extensive research has recently been conducted to find more cost-effective materials to replace silicon in PV systems. One of these materials is dye-sensitized solar cells (DSSCs) due to their low cost, facile fabrication, low toxicity, and high-power conversion efficiencies. Moreover, these cells have also proven to work under low light conditions [6]. A DSSC consist of a dye molecule with coated wide bandgap semiconducting layer, electrolyte (usually $I^{-} / I_{3}^{-}$redox couple), and transparent conducting oxide (TCO) film. The TCO is usually coated with mesoporous semiconductor $\mathrm{TiO}_{2}$ [7]. The dye molecule can include metal complexes and metal-free organic dyes. Research has recently focused on metal-organic frameworks (MOFs) as dye molecules due to their robustness, porosity, light-harvesting properties, thermal stability, and structural versatility. However, their insulating properties limit their usage [8-14]. In the past, $\mathrm{Ru}-$ based complexes held the top place for the highest efficiency in DSSCs of over $11 \%$ but were overtaken first in 2014 by zinc porphyrin compounds with an efficiency of $13 \%$, and soon afterwards in 2015 , by two co-sensitized metal-free organic dyes with an efficiency of $14.30 \%$ [15]. It was not just the efficiency of metal-free organic dyes that spurred researchers to focus more on them, but their low cost, simple synthetic procedures, high molar absorption coefficients and variable structure adjustability $[16,17]$. An organic dye usually consists of three fragments, namely, a donor, $\pi$-spacer, and an acceptor (D- $\pi$-A), where a push-pull system induces intramolecular charge transfer (ICT) from D to A via the $\pi$-spacer (Figure 1) [18-22]. Commonly used donor groups include triarylamine, starburst, carbazoles, indoles, phenoxides, phenothiazine and coumarins [21,2329 ], while commonly used $\pi$-spacer units include vinylene and thiophene groups [27,29], and commonly used as acceptor groups include cyanoacetic acid, rhodamine-3-acetic acid, barbituric acid, hydroxyl, phosphoric acid, cyanoacrylic acid and carboxylic acid [22,27,30]. ICT occurs due to sunlight hitting the compound and following a process that mimics photosynthesis [31]. Photophysicochemical properties of the dye will determine the efficiency of electron injection into the conduction band of $\mathrm{TiO}_{2}$. It is highly recommended that an ideal dye has a high diffusion constant $\left(D_{\pi}\right)$ to ensure minimal charge recombination. Successively, this leads to a large charge accumulation with a high charge collection efficiency $\left(n_{\text {collect }}\right)$ at the interface between the dye and the conduction band of $\mathrm{TiO}_{2}$. To achieve this, the dye should absorb in the near-infrared region (NIR), have a high fluorescence emission factor $\left(\Phi_{f}\right)$, high ground state molar absorption coefficient $\left(\varepsilon_{\mathrm{g}}\right)$, large oscillator strength $(f)$, and a high dipole moment [32]. The performance of a DSSC is dependent on a good light-harvesting efficiency $(L H E)$ from the dye and near quantitative collection (at the short circuit) [33]. As the ground state reduction potential of the conduction band $\left(E_{C B}\right)$ has a literature value of $-4.21 \mathrm{eV}$, it should be noted that for efficient transfer of photoelectrons from the 
dye into the conduction band of $\mathrm{TiO}_{2}$ semiconductor, the latter should have a higher reduction potential than the former. The electron injection rate depends on the difference between the LUMO of the dye and the conduction band edge $\left(\delta_{\mathrm{p}}\right)$ of the semiconductor (The smaller the difference, the faster the transfer). For a highly efficient transfer, the ideal difference should be $\leq$ $0.40 \mathrm{eV}$. This will subsequently lead to a faster photocurrent density in the cell. The energy level of the $I^{-} / I_{3}^{-}$redox couple should also lie above the HOMO level of the dye for electrons to be successfully regenerated back into the dye. The fact that the incident-photon-conversion-efficiency $(I P C E)$ is a function of the $L H E$, electron injection and charge collection efficiency prior to the injection makes it the key parameter to determine the efficiency of a dye [32]. Our study will analyze the photophysicochemical properties of compounds containing 1,3,4-oxadiazole $\pi$-spacer units (Figure 2 ). There have been some reports of compounds that contain this moiety as a $\pi$-spacer unit of a dye in DSSCs [34-36]. They are efficient electron transporters with high thermal stabilities and high photoluminescent quantum yields [37]. It is expected that highly energetic electrons will create large electron-hole separation distances, subsequently inducing small electron-hole overlaps. This will then cause them to diffuse more rapidly towards the anchoring segments, resulting in a high accumulation of charge that is more readily available for injection into the semiconductor's conduction band. Rapidly diffusing electrons will also be expected to regenerate more easily. As part of our ongoing research to theoretically assess the efficiencies of DSSCs containing novel metal-free organic dyes and provide plausible explanations for the observed trends, this study investigates the influence of selected moieties on the electron-hole charge separation distances in selected dye molecules. Furthermore, we explored correlations between the charge separation distance and other parameters such as electron-hole overlap, single-point excitation energies, electron densities across the anchoring segments, IPCE, dye regeneration, $L H E$, electron injection $\left(\Phi_{\text {inj }}\right)$ and $n_{\text {collect }}$. To the best of our knowledge, this is the first study that explores how electron-hole excitation processes determine the efficiencies of DSSCs. Herein, we provide the results from these analyses and their implications. 


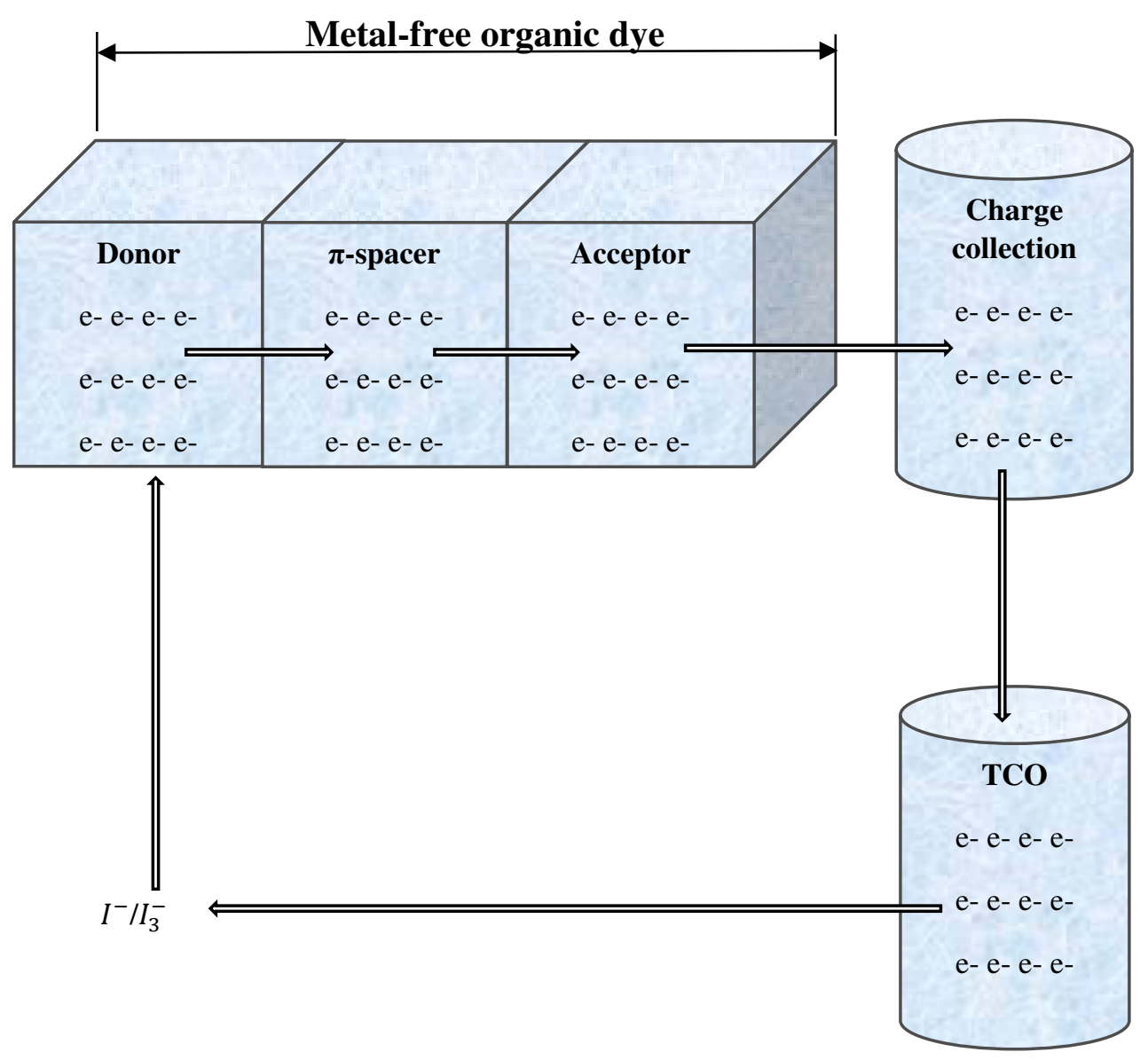

Figure 1: Mechanism for the excitation of photoelectrons within a metal-free organic dye and the regeneration of these photoelectrons back into the dye within a DSSC 

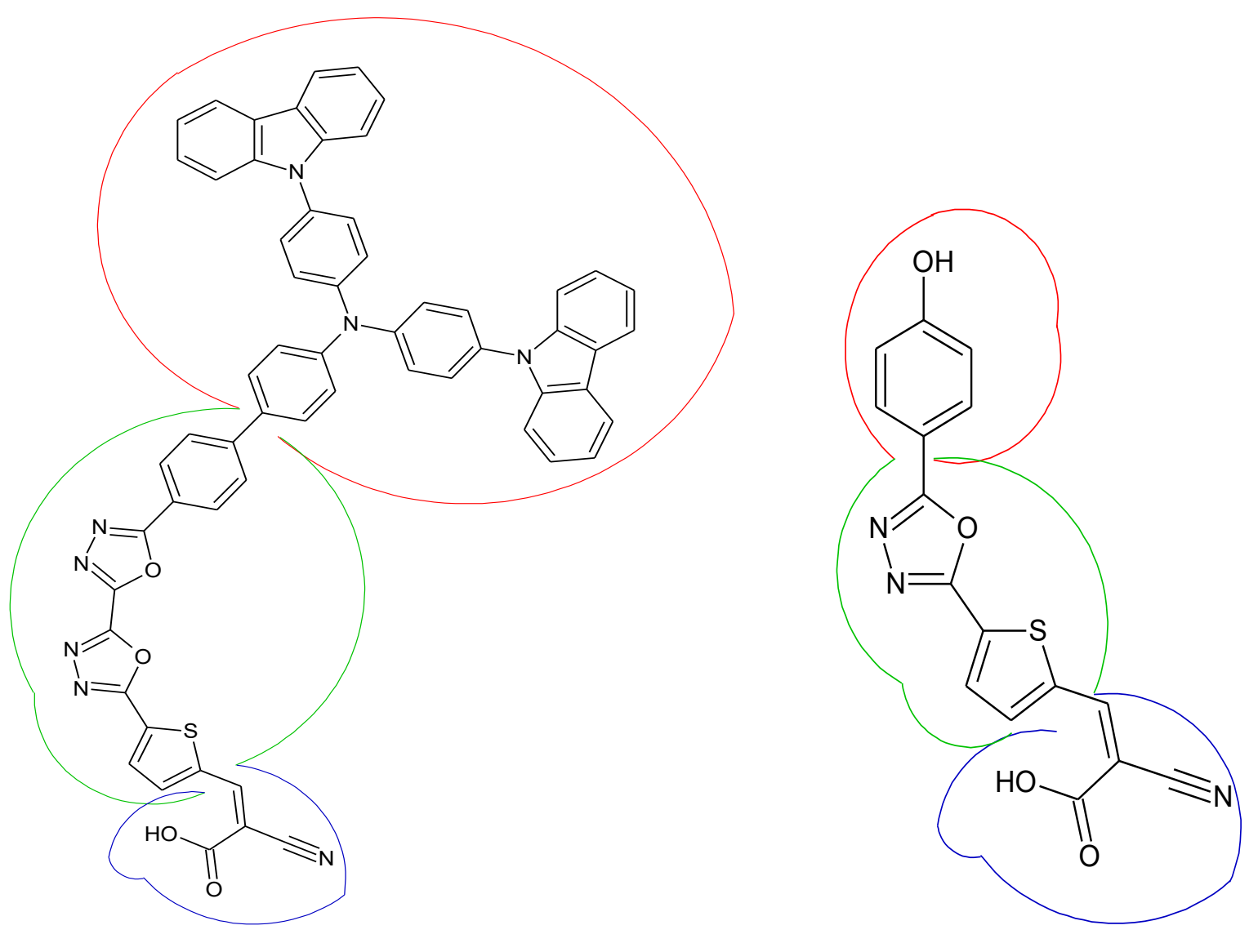

01

O2

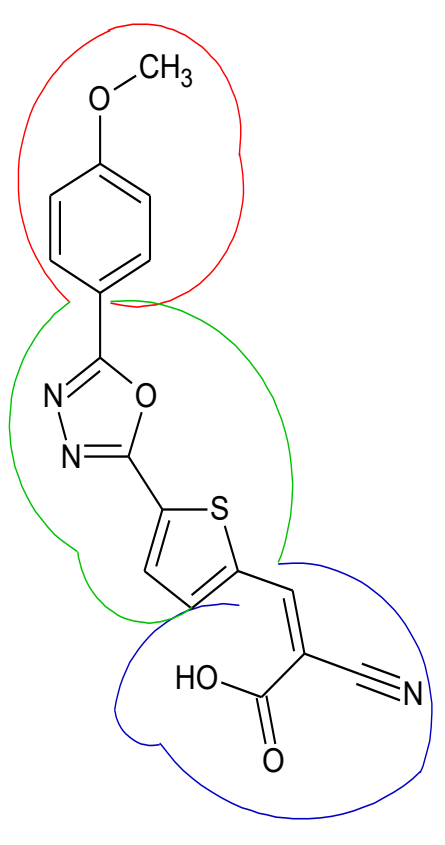

03

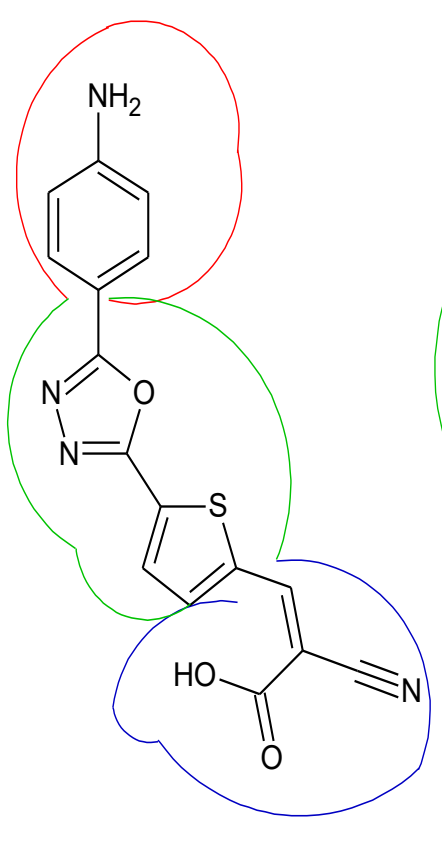

O4

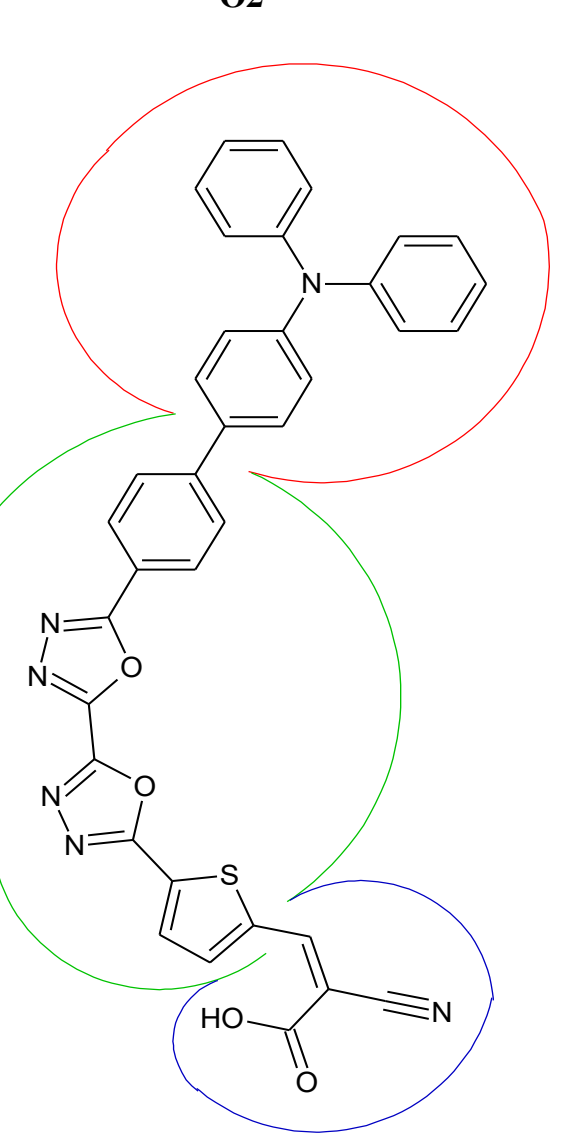

05 


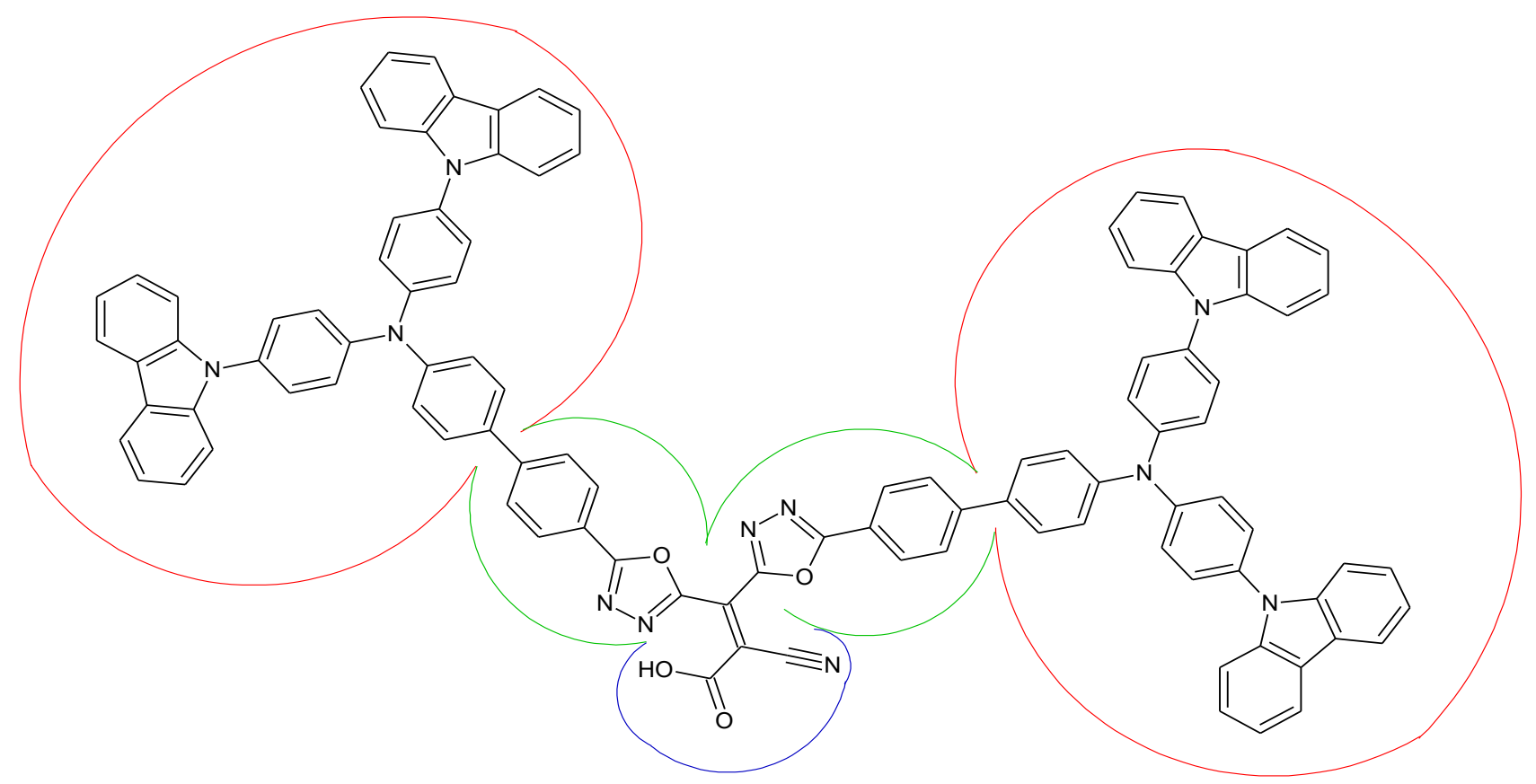

O6

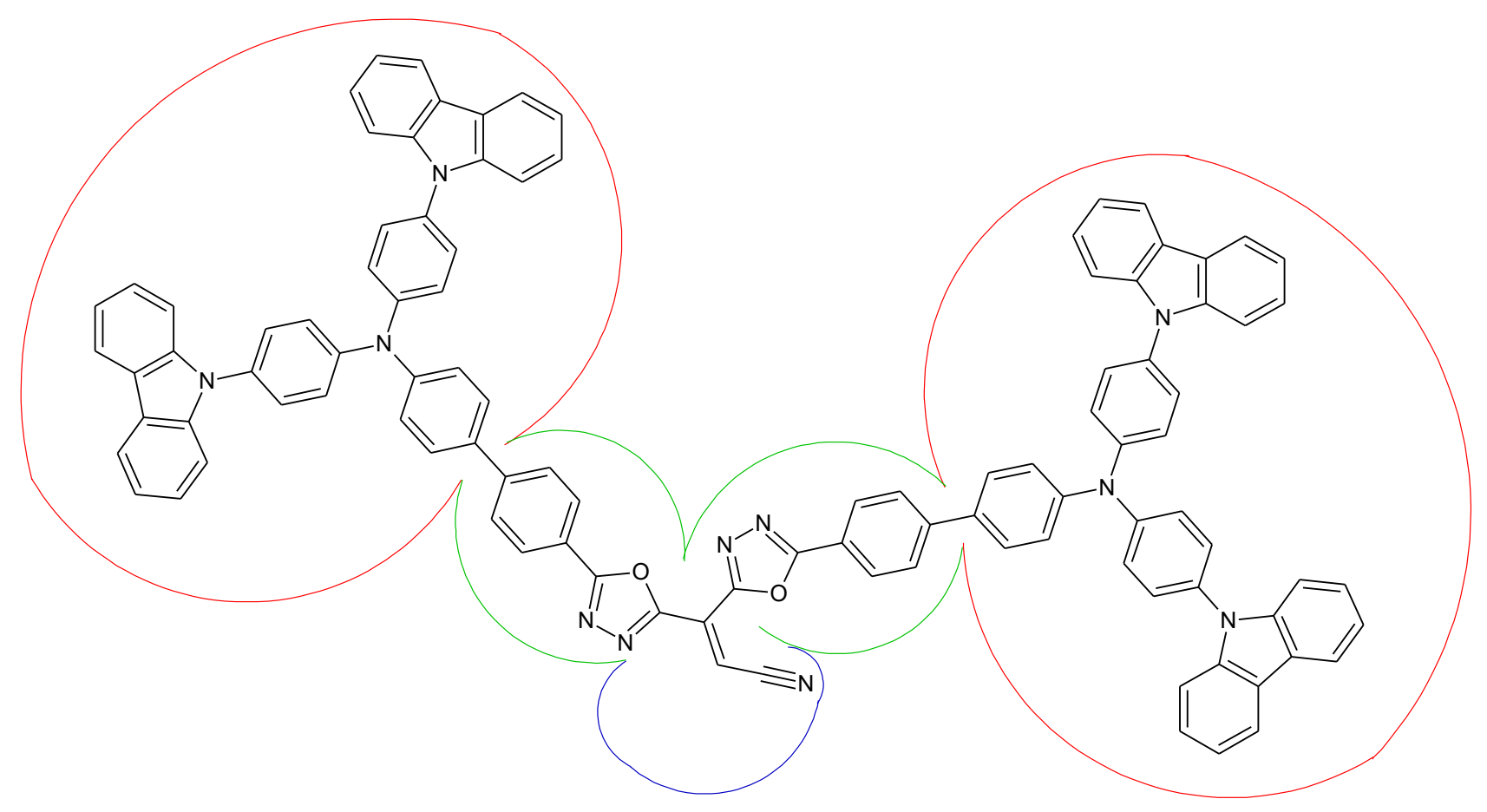

07

Figure 2: 1,3,4-oxadiazoles containing compounds that were evaluated for metal-free organic dyes through TD-

DFT calculations 


\section{Methodology}

\section{Computational details}

All calculations were carried out using Gaussian 09 rev E01 software [38]. The geometries of all molecules were optimized in the gas phase for both DFT and TD-DFT calculations. All structures were optimized at the M06-2x/6-31G(d,p) level of theory. The optimization process was carried out in conjunction with frequency calculations to verify that minimum energies were obtained for the

\section{Results and discussion}

\section{Optimized geometry}

The predicted property of a molecule depends heavily on the accuracy of its optimized structure. A series of optimization steps are required for each structure before reaching its minimum energy configuration (Figure S1)-conventionally known as the optimized structure. The optimized structures were validated as minima or transition state structures by performing frequency calculations. As any structure with one imaginary frequency (i.e. negative vibrational frequency) is denoted by a transition state, higher-order saddle points are assigned to structures with two or more imaginary frequencies. Hence, it is generally accepted that a structure that has reached its minimum energy should not have a negative frequency, and hence, are fully optimized. However, in the case of very large floppy molecules, negative vibrational frequencies from $10-100 \mathrm{~cm}^{-1}$ can be ignored [40]. structures. Using their optimized structures as input, we carried out TD-DFT calculations on the organic dyes labelled as 01-07 (Figure 2) in the gas phase and their frontier molecular orbital natures (FMO) were analyzed with multiwfn software [39] by dividing their structures into D- $\pi$-A fragments to study their ICT at the same level of theory. In addition, molecular orbital structures, and energies of their HOMO and LUMO were also obtained from an analysis of the optimized structures.

\section{Coulomb attractive energy and electron excitation analyses}

The efficiency of a DSSC depends on the electrooptical properties and electron-hole pair dissociation potential of the metal-free organic dye. As Coulombic forces exist between electron-hole pairs, binding (Coulomb attractive) energy hold the electron to the hole. The low dielectric constant of organic molecules causes a strong Coulombic force between the electron-hole pair, leading to high binding energy, inversely proportional to the dissociation energy. The latter causes the electron to depart from the hole. Equation 1 gives the relation between binding energy, band gap energy and single point excitation energy.

$E_{b}=E_{(\text {LUMO-HOMO })}-E_{(S I-S O)}$

Where $E_{b}$ is the binding energy, which can also be classified as the estimation of dissociation energy of excitation, $E_{(\text {LUмо-номо })}$ is the band gap energy, and $E_{(S I-S O)}$ is the single point excitation energy between the ground and the excited state. For an electron to get successfully excited to the LUMO level, it must possess positive single point energy. This also separates it from its hole [41]. The ionization potential (IP) and electron affinity (EA) 
can predict the electron-hole transport barrier. The former can be calculated using equation 2 .

$\mathrm{IP}=-E_{\text {НОМO }}$

The latter can be calculated through equation 3

EA

$$
=\quad-E_{L U M O}
$$

Previously, it was believed that a narrow $E_{(L U M O)}$ (номо) energy gap means higher IP [42]. However, a recent study by Bulat et al. revealed that the energies of electrons in specific atomic or molecular orbitals play significant roles in excitation processes. Electrons occupy specific sites within the space of a molecule irrespective of the molecular orbitals that they may occupy. If an orbital $\varphi_{\mathrm{i}}$ has an electron density $\rho_{\mathrm{i}}(r)$ and energy $\varepsilon_{\mathrm{i}}$, then the total electronic density is $\rho(r)$, and the average orbital energy is

$$
\bar{\varepsilon}(r) \quad=\quad \frac{\sum \rho_{i}(r) \varepsilon_{i}}{\rho(r)}
$$

The summation of Eq. (4) over all the occupied orbitals is

$\overline{I(r)} \quad=\quad \frac{\sum \rho_{i}(r)\left|\varepsilon_{i}\right|}{\rho(r)}$

Where $\overline{I(r)}$ is the average local ionization energy at $\boldsymbol{r} . \overline{I(r)}$ is the average energy required to remove an electron at point $\boldsymbol{r}$ in the space of an atom or molecule. The least tightly held electrons within atomic or molecular space are also the most energetic and correspond to the lowest $\overline{I(r)}$ values. Like Fukui's reasoning, these are the likely sites for electrophilic substitution, protonation, and free radical attack. $\overline{I(r)}$ were shown to be related to other critical molecular properties such as kinetic energy density and local temperature, local polarizability/hardness, electronegativity, atomic shell structure and the electrostatic potential. When
Bulat et al. studied the $\overline{I(r)}$ of twelve molecules, they observed that only five amongst the twelve contained electrons that occupied the HOMO [43]. As electrons get excited, they are distributed unsymmetrically, causing an increase in the polarity in the excited state. The polarity of the molecule influences its geometry, which can be measured by the dipole moment. Thus, the symmetry of the molecule is inversely proportional to the dipole moment. For an electron to get excited, it must absorb a photon. The probability for a photon to be absorbed is proportional to its oscillator strength $(f)$ [41]. Since the excited state is less stable than the ground state, electrons will return to the latter. Other than 07, all the compounds in Table 1 yield positive single point excitation energies. Thus, within most compounds, electrons will get excited. The highest single point energy in $\mathbf{O 5}$ indicates that an electron will get excited more easily than the other compounds under study. However, its $f$ is less than that of $\mathbf{O 2}$. The low dipole moment in $\mathbf{O 4}$ means that it is highly symmetrical, and upon excitation, an electron will more easily relax to the ground state than within the other compounds.

As the dipole moment indicates the solubility of a molecule in polar solvents [41], it is expected that $\mathbf{O 4}$ will be the least soluble while 01 will be the most soluble. Upon dissolution, an increase in the wavelengths of these compounds is expected due to interaction with solvent molecules [41]. Although O6 does not contain the highest IP, it does have the highest EA. This confirms Bulat et al. findings [43]. However, its $\varepsilon_{\text {abs }}$ is very low compared to other dyes. As all the LUMO energies lie above the literature value of the conduction band edge of $\mathrm{TiO}_{2}(-4.21 \mathrm{eV})$ [32], each compound has the potential to successfully transfer electrons into the conduction band edge once excited. As their HOMO energies lie below the literature value of 
the HOMO energy $(-4.6 \mathrm{eV})$ of $I^{-} / I_{3}^{-}[32]$, their electrons can also be successfully regenerated back into the dye. Other than $\mathbf{O 6}$ and $\mathbf{0 7}$, none of the compounds absorbs in the visible and Near-infrared

(NIR)

regions.

Table 1: Electron excitation parameters of each molecule

\begin{tabular}{|c|c|c|c|c|c|c|c|c|c|c|c|}
\hline compound & $\begin{array}{l}\underline{\mathrm{HOMO}} \\
\underline{(\mathrm{eV})}\end{array}$ & $\begin{array}{l}\underline{\text { LUMO }} \\
\underline{(\mathrm{eV})}\end{array}$ & $\begin{array}{l}\underline{E}_{(L U M O-H O M O)} \\
\underline{(\mathrm{eV})}\end{array}$ & $\begin{array}{l}\underline{E_{b}} \\
\underline{(\mathrm{eV})}\end{array}$ & $\begin{array}{l}\underline{E}_{(S I-S 0)} \\
\underline{(\mathrm{eV})}\end{array}$ & $\begin{array}{l}\text { Dipole } \\
\text { moment } \\
\underline{\text { (Debye) }}\end{array}$ & $f$ & $\begin{array}{l}\underline{\varepsilon_{a b s}} \\
\underline{\left(\mathrm{L} \cdot \mathrm{mol}^{-1} \cdot \mathrm{cm}^{-1}\right)}\end{array}$ & $\begin{array}{l}\underline{\lambda_{\max }} \\
\underline{(\mathrm{nm})}\end{array}$ & $\begin{array}{l}\underline{\mathrm{IP}} \\
\underline{(\mathrm{eV})}\end{array}$ & $\begin{array}{l}\underline{\text { EA }} \\
\underline{(\mathrm{eV})}\end{array}$ \\
\hline 01 & \begin{tabular}{|l|}
-6.95 \\
\end{tabular} & -2.59 & 4.36 & 3.72 & 0.64 & 7.48 & 0.545 & 27692 & 337 & 6.95 & 2.59 \\
\hline $\mathbf{O 2}$ & \begin{tabular}{|l|}
-7.37 \\
\end{tabular} & -2.15 & 5.22 & 4.50 & 0.72 & 2.81 & 1.030 & 41649 & 356 & 7.37 & 2.15 \\
\hline $\mathbf{O 3}$ & \begin{tabular}{|l|}
-7.23 \\
\end{tabular} & -2.17 & 5.06 & 4.17 & 0.89 & 5.73 & 0.853 & 34505 & 360 & 7.23 & 2.17 \\
\hline O4 & \begin{tabular}{|l|}
-7.59 \\
\end{tabular} & -2.12 & 5.47 & 4.23 & 1.23 & 0.91 & 0.675 & 28404 & 325 & 7.59 & 2.12 \\
\hline 05 & \begin{tabular}{|l|}
-7.39 \\
\end{tabular} & -2.45 & 4.94 & 3.37 & 1.57 & 6.66 & 0.782 & 37278 & 325 & 7.39 & 2.45 \\
\hline O6 & \begin{tabular}{|l|}
-6.69 \\
\end{tabular} & -2.95 & 3.74 & 3.43 & 0.31 & 3.18 & 0.042 & 3522 & 429 & 6.69 & 2.95 \\
\hline O7 & \begin{tabular}{|l|}
-7.06 \\
\end{tabular} & -2.58 & 4.48 & 4.65 & -0.17 & 5.12 & 0.380 & 15470 & 496 & 7.06 & 2.58 \\
\hline
\end{tabular}

\section{Photovoltaic parameters}

As IP and EA measure the injection abilities of holes and electrons, the greater EA becomes, the more electrons are received at the acceptor fragment (For a detailed discussion of the atombased electron-hole analysis, see ESI) [42,44]. From the anchoring groups of the acceptor fragment, electrons diffuse into the $\mathrm{TiO}_{2}$ conduction band via a diffusion constant $D_{\pi}$, which can be calculated using Stoke's equation 6 .

$D_{\pi}=\frac{K_{B} T}{6 \pi \eta r_{d y e}}$

Where $K_{B}$ is Boltzman's constant, $\eta=$ the viscosity of the medium, which can be assumed to be $\mathrm{He}$ at $300 \mathrm{~K}\left(20.0 \times 10^{-6} \mathrm{Pas}\right)$ in this case since the investigations were performed in the gas phase, $N_{A}$ $=$ Avogadro's number and $r_{d y e}=$ the molecular radii of the dye. The latter can be obtained from equation 7

$r_{d y e}=a=\sqrt[3]{\frac{3 M}{4 \pi \rho N A}}$ where $a=$ Onsager cavity radii, $M=$ the molecular weight of the dye, $\rho=$ the density of $\mathrm{He}$ gas $\left(9.00 \times 10^{-2} \mathrm{~kg} \cdot \mathrm{m}^{-3}\right)$ at STP and $N_{A}=$ Avogadro's Number. As stated earlier, the diffusion constant plays a key role in minimizing charge recombination during excitations (electrons that diffuses more rapidly will have a lower probability for charge recombination) [22]. The low molecular weights of $\mathbf{O 2}$ and $\mathbf{O 4}$ causes them to display the highest $D_{\pi}$ (Table 2). Thus, due to their small sizes compared to the other compounds, their photoelectrons will diffuse more rapidly into the conduction band of $\mathrm{TiO}_{2}$. As electrons diffuse towards the conduction band of $\mathrm{TiO}_{2}$, a charge builds up and is collected. The efficiency of this collection is called the charge collection efficiency $\left(n_{\text {collect }}\right)$, which can be obtained through equation 8

$n_{\text {collect }}$

$$
=
$$$$
\frac{D_{\pi}}{\left(\delta_{p}\right)^{2}}
$$

where $\delta_{p}$ is the potential difference between the LUMO of the dye and the conduction band of $\mathrm{TiO}_{2}$. The $n_{\text {collect }}$ measures the availability of electrons 
before injection [22]. The high LUMO excitedstate energy in $\mathbf{O 3}$ causes it to accumulate more charge across its $\eta_{\text {collect }}$ than the other compounds. As a low potential difference between the LUMO excited state energy and the conduction band of $\mathrm{TiO}_{2}$ exist due to the low excited state energy of the former, low charge build-up in $\mathbf{0 6}$.

Light harvesting efficiency ( $L H E)$, which measures the spectral distribution of sunlight that is being absorbed onto the molecule, can be expressed as

LHE$$
=
$$

Where $f$ is the oscillator strength of the dye molecules. From equation 9, we can deduce that a large oscillator strength will yield a high $L H E$. Thus, the large $f$ in $\mathbf{O 2}$ causes it to display a large $L H E$. If the excited electron injection efficiency $\left(\Phi_{i n j}\right)$ of the dye is assumed to be equal to the fluorescence emission factor $\left(\Phi_{f}\right)$, then we can define the latter as in equation 10

$\Phi_{f} \quad=\quad \frac{I \varepsilon_{e m}}{I \varepsilon_{a b s}}$

where $I \varepsilon_{e m}$ and $I \varepsilon_{a b s}$ are the integrated emission and absorption coefficients, respectively, correspond to the areas under the emission and absorption spectra. The former can be obtained through the extrapolation of the absorption spectra [22]. Due to a small difference between the absorption and emission spectrum in $\mathbf{O 3}$, a large $\Phi_{i n j}$ is displayed in this compound. Thus, more photoelectrons can be transferred from the LUMO of this compound into the conduction band of $\mathrm{TiO}_{2}$ than the other compounds. Conversely, a small $\Phi_{i n j}$ is observed in O6 due to large differences between absorption and emission spectra within this compound that arises from possible photo collisions [43]. The IPCE can then be obtained through equation 11
As mentioned earlier, IPCE is the key factor determining the efficiency at which the dye can inject photoelectrons into the conduction band of $\mathrm{TiO}_{2}$ [22]. The large $\Phi_{i n \mathrm{j}}$ in $\mathbf{O 3}$ causes a large IPCE, while the small $\Phi_{i n j}$ in $\mathbf{O 6}$ causes a very small IPCE. An electron can be efficiently transferred to the first excited state if it has a long lifetime $(t)$, which is denoted by the equation

$$
t
$$$$
=\quad \frac{1.499}{f \times E^{2}}
$$

where $f$ is the oscillator strength, and $E\left(\mathrm{~cm}^{-1}\right)$ is the excitation energy of the different electronic states [44]. The long $t$ for the excited state in $\mathbf{O 6}$ means that electrons can be more efficiently transferred to a higher energy level within this compound than the other compounds.

The free energy obtained from the electron injection $\left(\Delta G_{\text {inject }}\right)$ can be expressed as

$\Delta G_{\text {inject }}=E_{O x}^{\text {dye* }}=E_{O x}^{\text {dye }}-E_{00}$

where $E_{O x}^{d y e}$ is the oxidation potential energy of the dye in the ground state, while $E_{00}$ is the electronic vertical transition energy corresponding to $\underline{\lambda}_{\max }$ [44].

The regeneration of free energy $\left(\Delta G_{d y e}^{\text {regen }}\right)$ is a significant factor that can affect the photoelectric conversion efficiency and can be expressed as

$\Delta G_{\text {dye }}^{\text {regen }}=E_{\text {redox }}^{\text {electrolyte }}-E_{\text {ox }}^{\text {dye }}$ (14)

where a large $\Delta G_{\text {dye }}^{\text {regen }}$ can promote dye regeneration and increase the $J_{S C}[22,45]$. As 04 displayed the highest $\Delta G_{d y e}^{r e g e n}$, its photoelectrons will be more easily regenerated. 
Table 2: Photovoltaic parameters of compounds that were tested as organic dyes

\begin{tabular}{|l|l|l|l|l|l|l|l|l|l|}
\hline Compound & $\begin{array}{l}\boldsymbol{r}_{\text {dye }}(\mathrm{m}) \\
\left(1 \times 10^{-7}\right)\end{array}$ & $\begin{array}{l}\boldsymbol{D}_{\boldsymbol{\pi}} \\
\left(1 \times 10^{-11}\right)\end{array}$ & $\boldsymbol{\delta}_{\boldsymbol{p}}$ & $\begin{array}{l}\boldsymbol{\eta}_{\text {collect }} \\
\left(1 \times 10^{-12}\right)\end{array}$ & $\mathbf{L H E}$ & $\boldsymbol{t}(\mathrm{s})$ & $\boldsymbol{\Phi}_{\text {inj }}$ & $\begin{array}{l}\text { IPCE } \\
\left(1 \times 10^{-38}\right)\end{array}$ & $\begin{array}{l}\Delta \boldsymbol{G}_{\text {dye }}^{\text {regen }} \\
(\mathrm{eV})\end{array}$ \\
\hline $\mathbf{O 1}$ & 1.62 & 6.73 & -6.08 & 1.68 & 0.715 & 0.212 & 0.795 & 1.21 & 2.35 \\
\hline $\mathbf{O 2}$ & 1.15 & 9.51 & -5.64 & 2.99 & 0.907 & 0.120 & 1.00 & 2.33 & 2.77 \\
\hline $\mathbf{O 3}$ & 1.16 & 9.47 & -5.66 & 2.96 & 0.860 & 0.148 & 1.00 & 2.55 & 2.63 \\
\hline $\mathbf{O 4}$ & 1.15 & 9.51 & -5.61 & 3.02 & 0.789 & 0.154 & 0.961 & 2.29 & 2.99 \\
\hline $\mathbf{O 5}$ & 1.41 & 7.73 & -5.94 & 1.51 & 0.835 & 0.136 & 0.849 & 1.55 & 2.79 \\
\hline $\mathbf{O 6}$ & 1.89 & 5.76 & -6.44 & 1.39 & 0.0922 & 4.56 & 0.480 & 0.0615 & 2.09 \\
\hline $\mathbf{O 7}$ & 1.54 & 7.07 & -6.07 & 1.92 & 0.583 & 0.633 & 0.996 & 1.11 & 2.46 \\
\hline
\end{tabular}

\section{Fragment-based electron-hole analysis}

After dissecting each molecule into atoms to establish the concentration of electrons excited to the anchoring groups (see ESI), an evaluation was performed on the fragment contribution to electronhole analysis (Figures 3 and 4). In Figure 3, the values on the right-hand side of the y-axis represent the hole, electron and overlap contributions, where purple indicates the least contribution, while red indicates the highest contribution. The numbers 1 , 2 and 3 in the $\mathrm{x}$-axis represents the donor, $\pi$-spacer, and acceptor fragments, respectively. These analyses were also performed for the first excited state. A loss of $1.12 \%$ in $\mathbf{O 1}$ is observed for the donor fragment during excitations as the charge is transferred to the second fragment (Table S8). At the second fragment, $35.68 \%$ of electrons are lost during excitations, which is then transferred to the third fragment. About $36.52 \%$ of electrons is then delocalized to the third fragment due to these excitations. Larger electron-hole contributions for the donor and $\pi$-spacer groups than their electron contributions, as well as smaller overlap analyses for the acceptor group than its electron contributions, confirm this. This also agrees with the atom-based electron-hole analyses. Although to a lesser degree, a similar scenario is observed in O2, which also agrees with the atom-based electron-hole analyses. The same trend holds for the rest of the compounds as $\mathbf{O 1}$ and $\mathbf{0 2}$ (Tables S11-S14) and agrees with our atom-based electronhole analyses as well. 


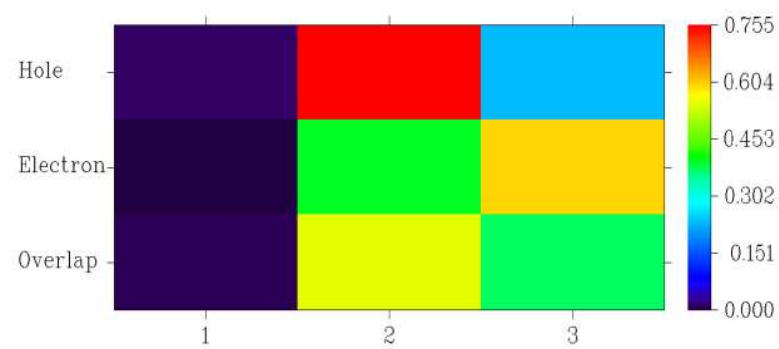

01

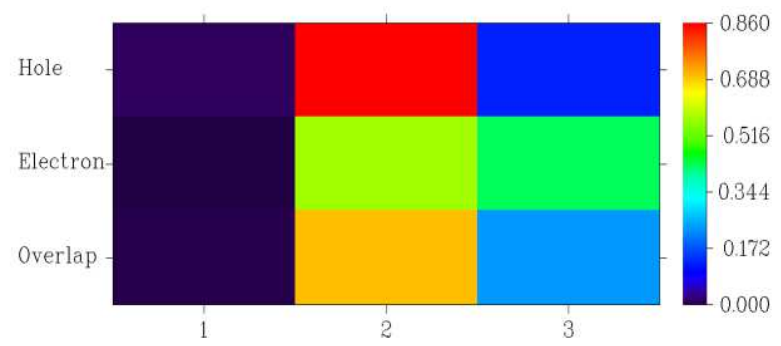

02

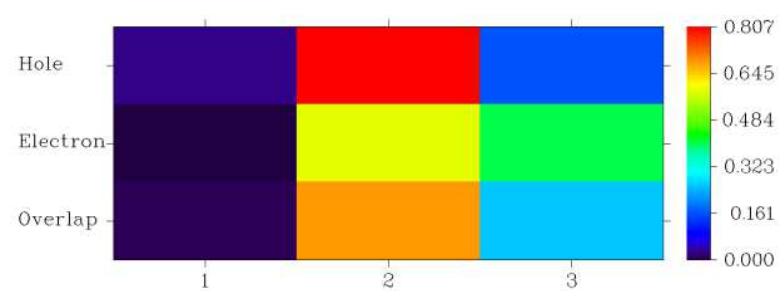

$\mathbf{0 3}$

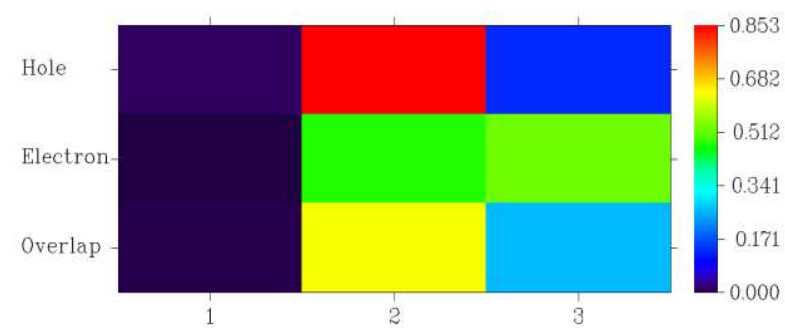

04

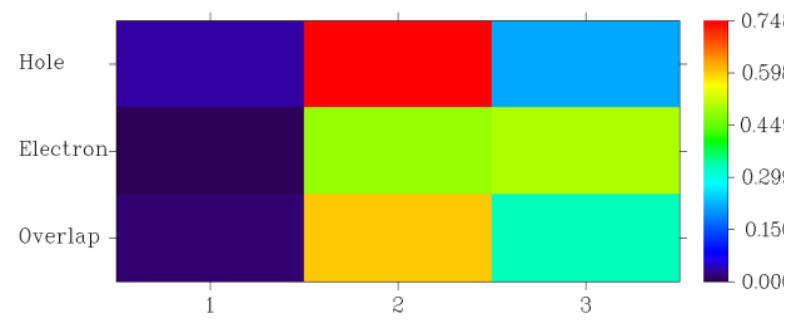

05

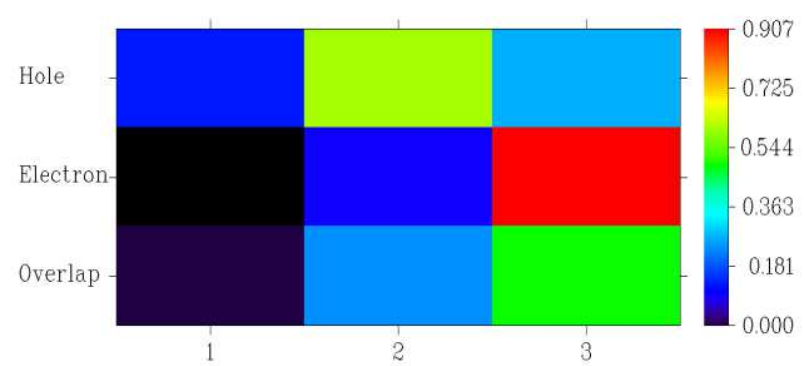

O6

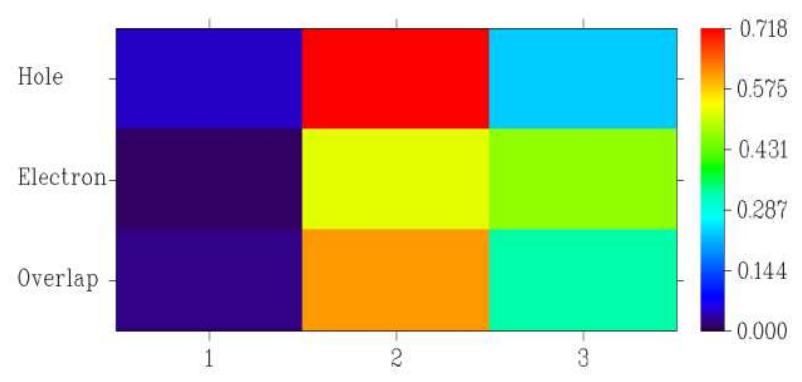

07

Figure 3: Electron-hole overlaps for each fragment within each molecule

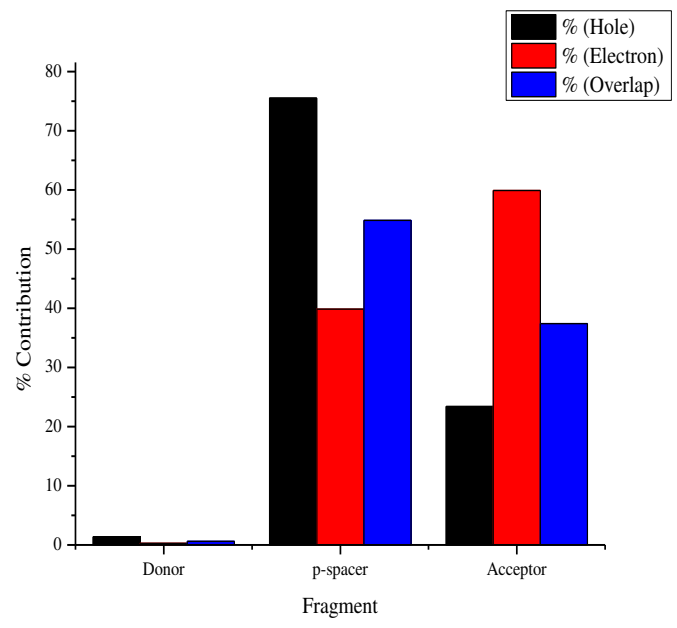

01 


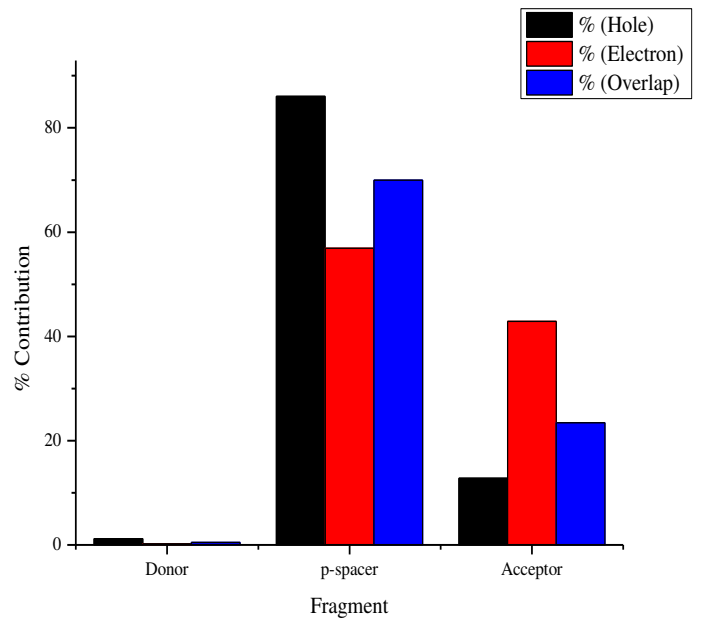

O2

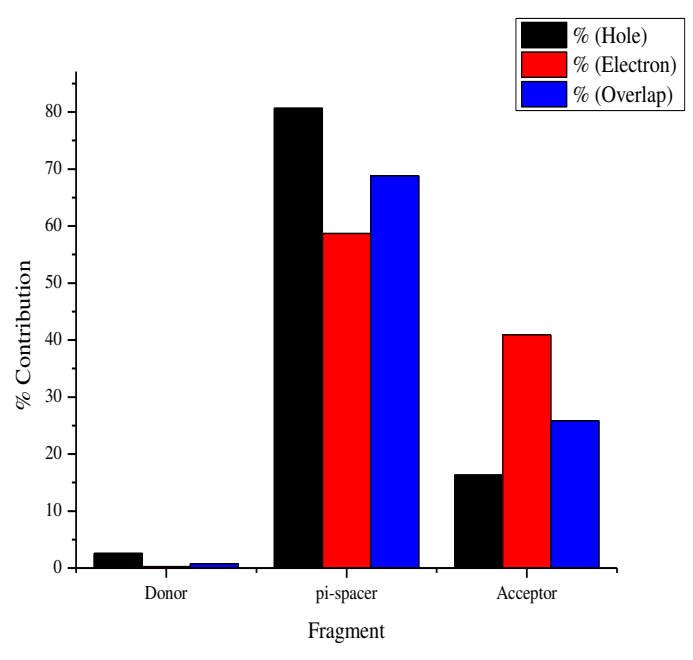

03

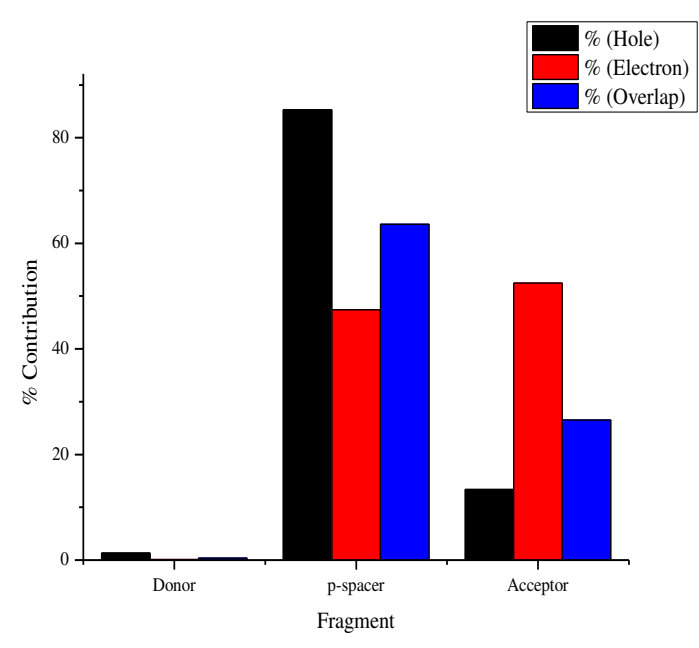

O4

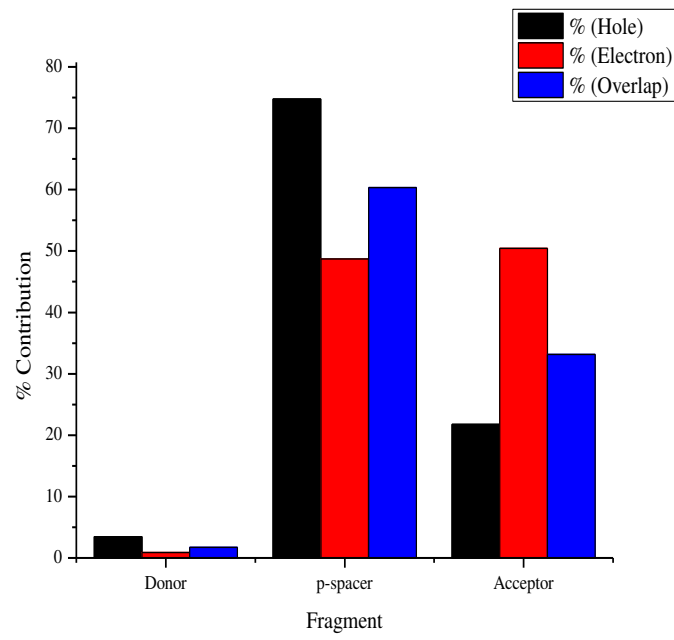

05

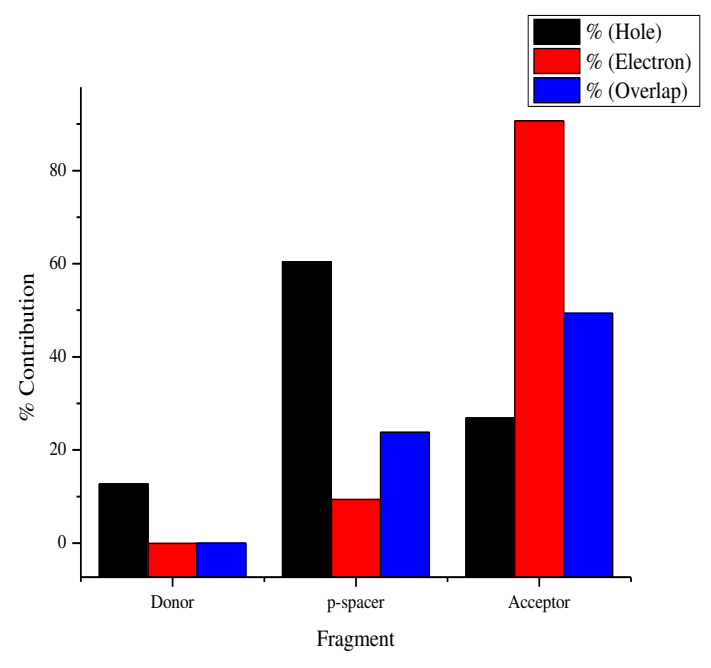

06

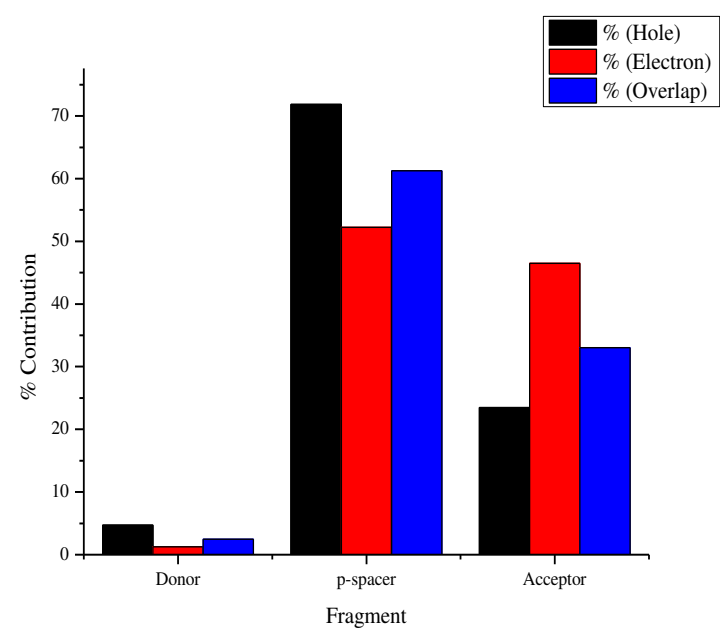


Figure 4: Electron-hole contribution to each fragment within each compound

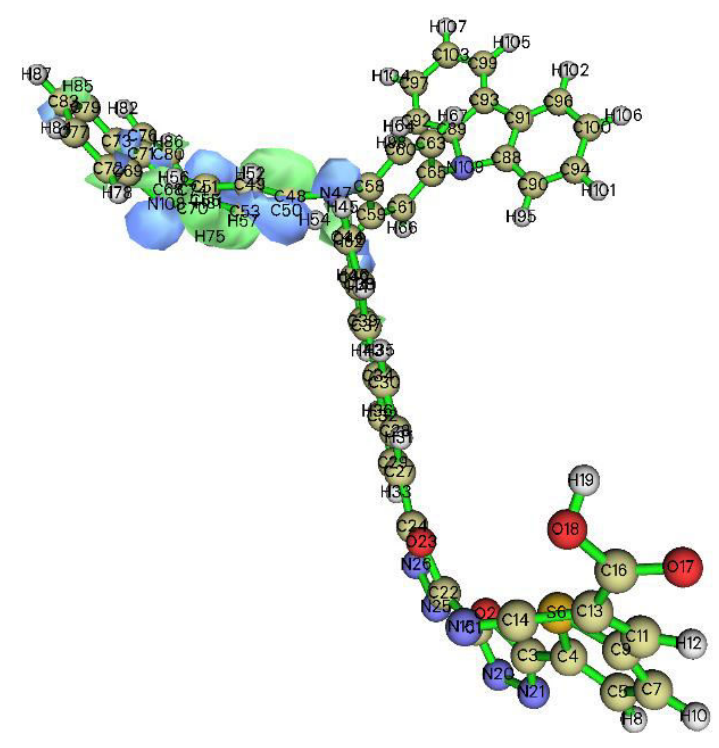

HOMO of $\mathbf{O 1}$

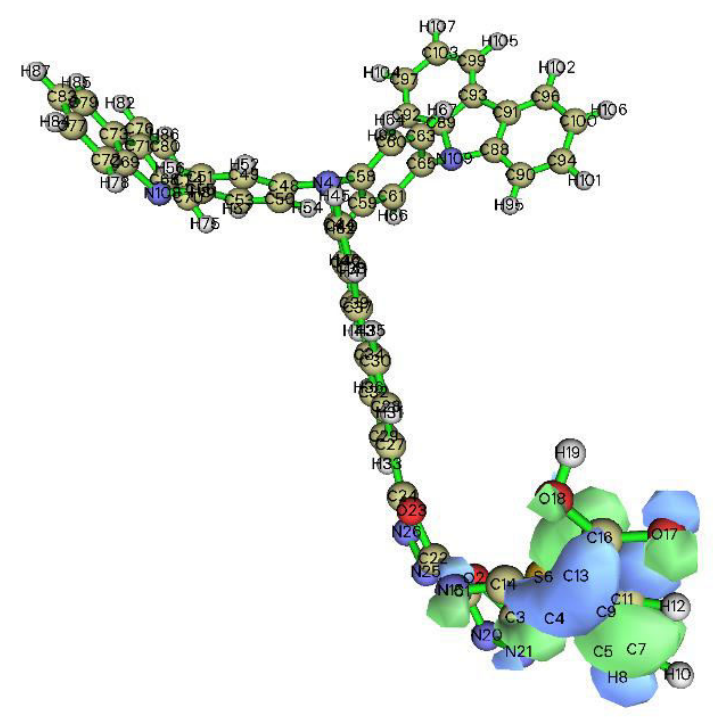

LUMO of $\mathbf{0 1}$

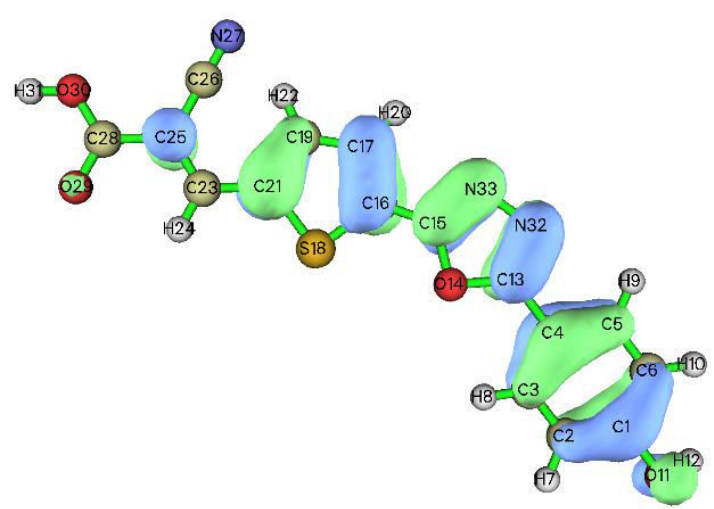

HOMO of $\mathbf{O 2}$

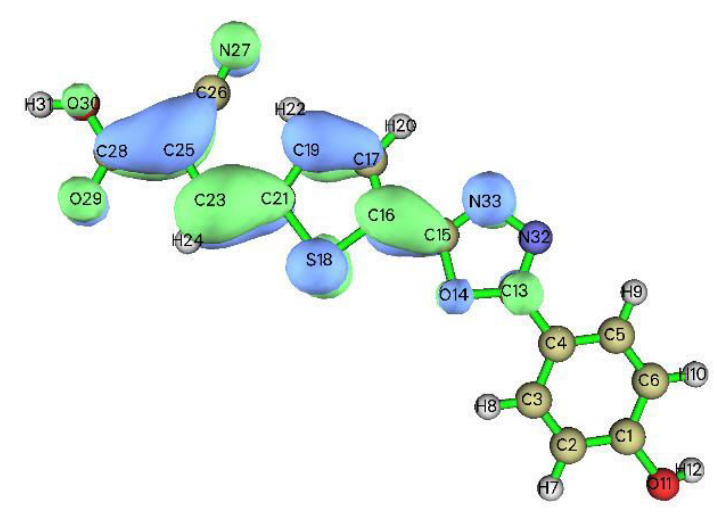

LUMO of $\mathbf{O 2}$

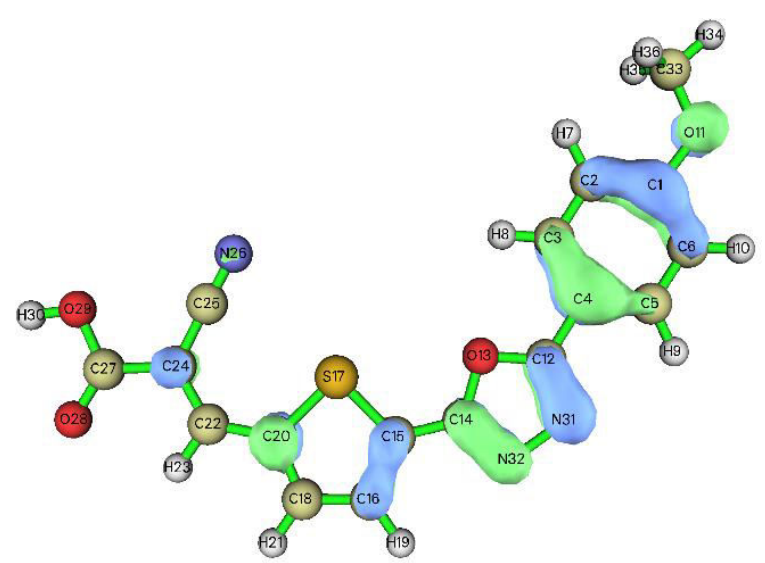

HOMO of $\mathbf{O 3}$ 


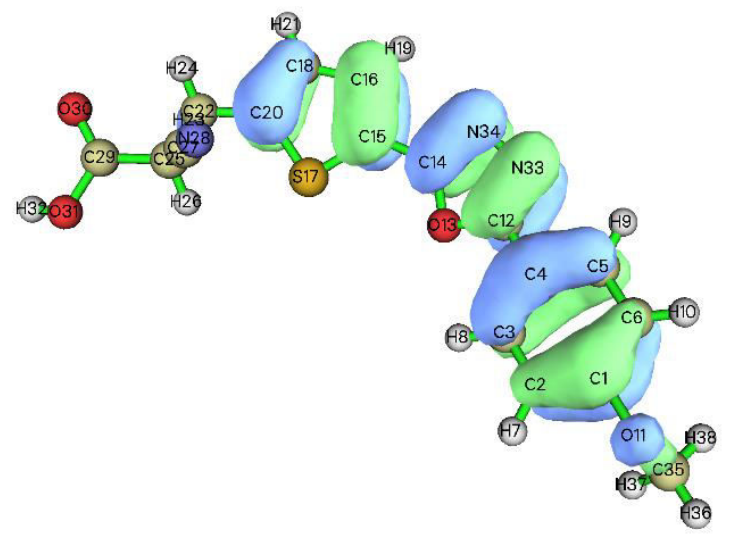

LUMO of $\mathbf{O 3}$

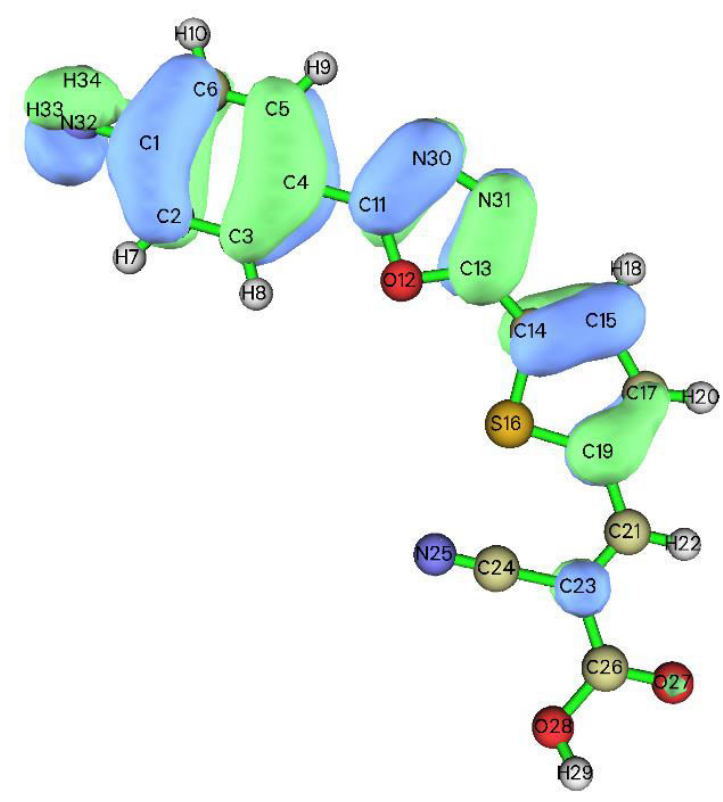

HOMO of $\mathrm{O4}$

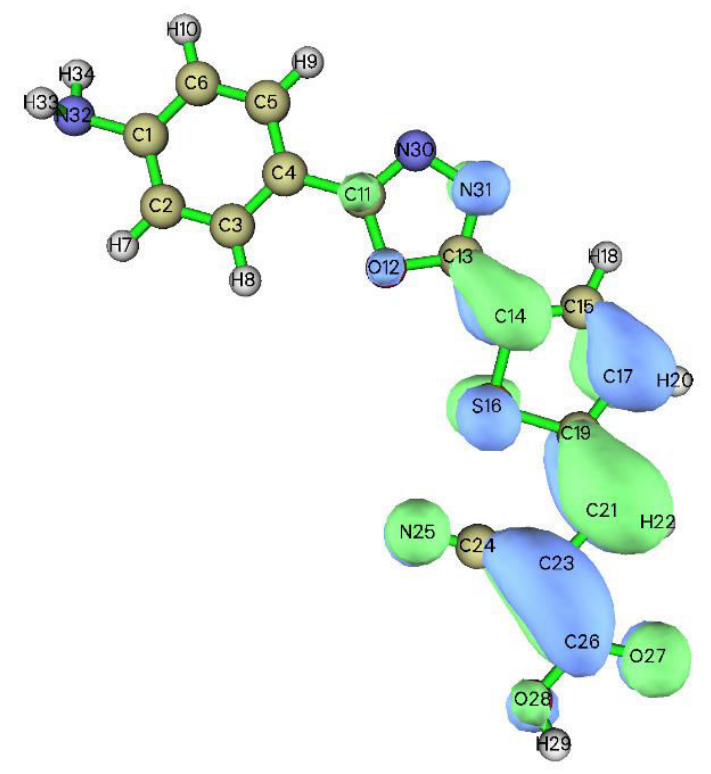

LUMO of $\mathbf{O 4}$

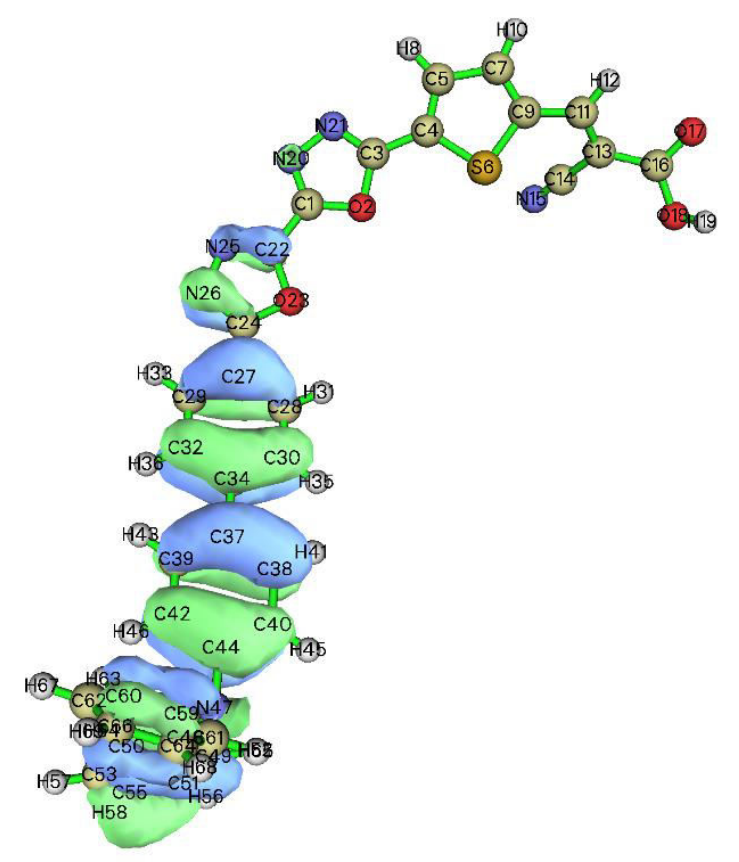

HOMO of $\mathbf{O 5}$ 

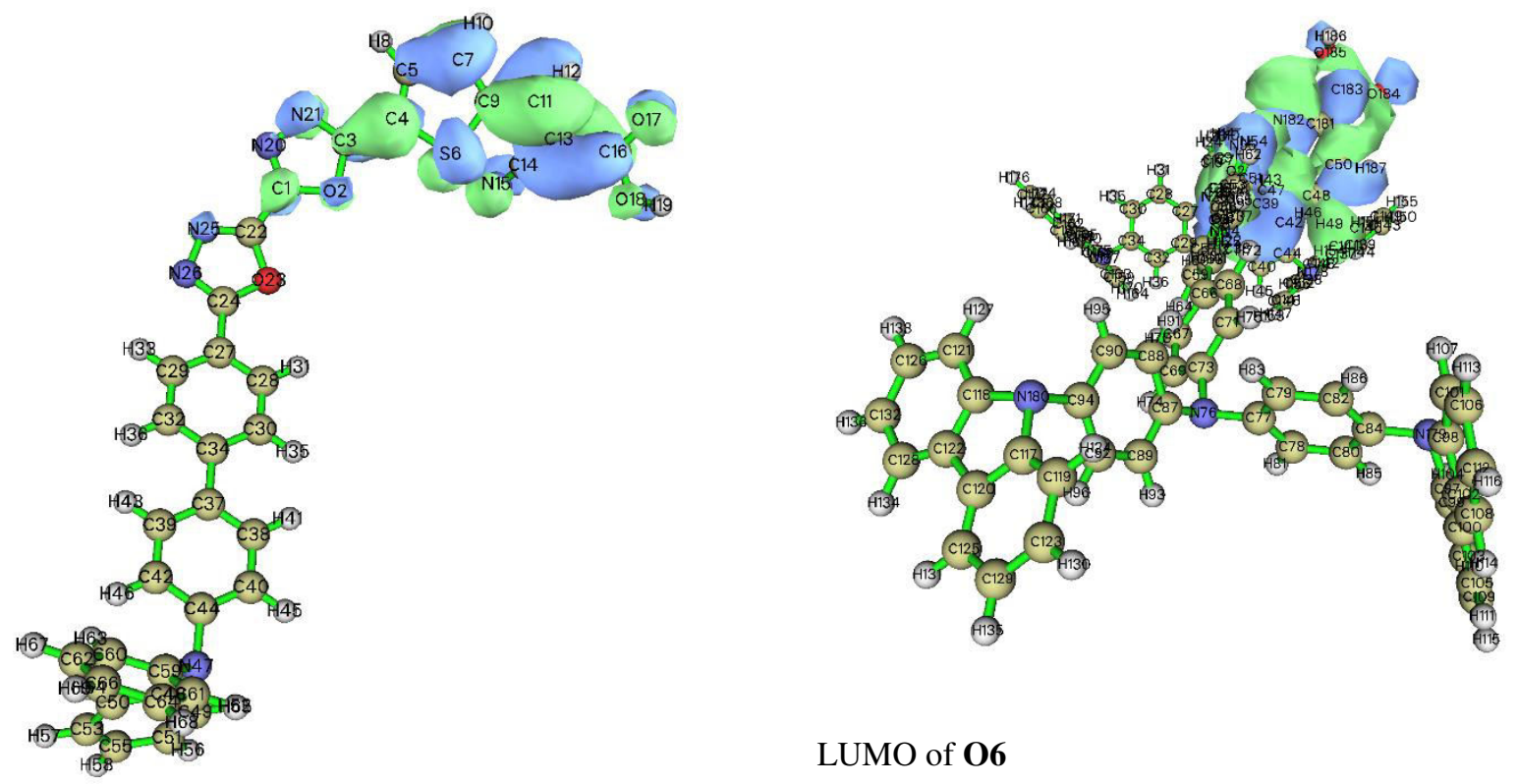

LUMO of $\mathbf{0 5}$

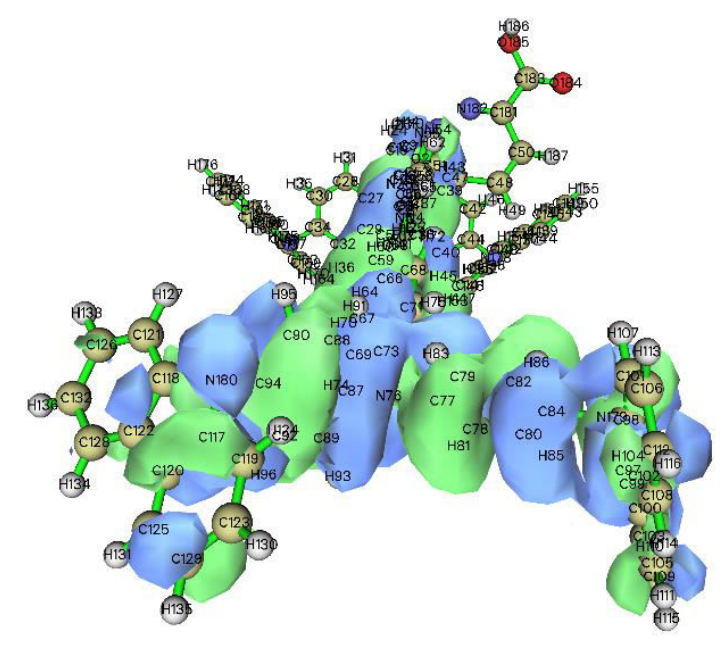

LUMO of $\mathbf{O 6}$

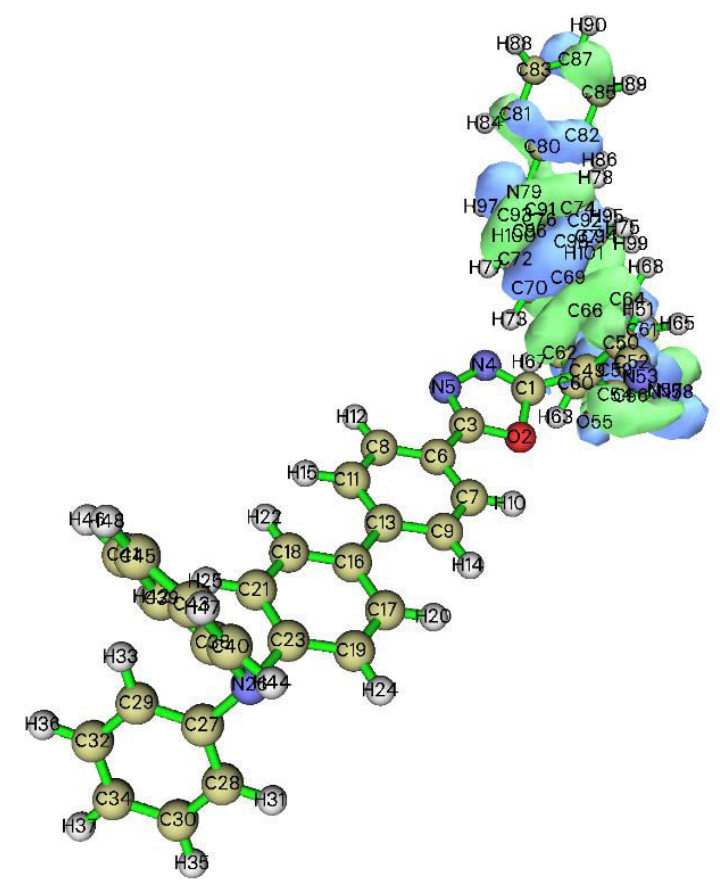

HOMO of $\mathbf{0 7}$ 


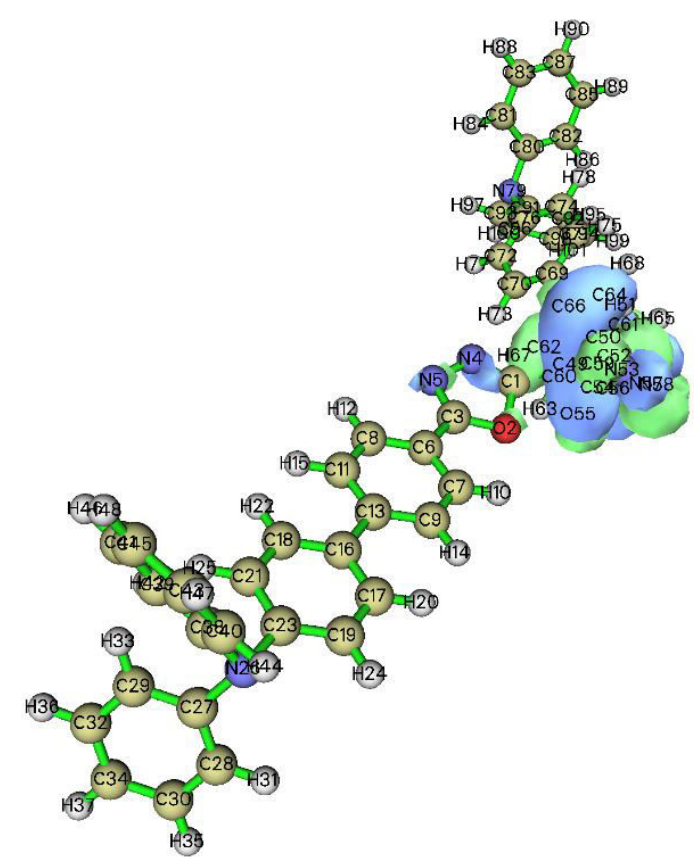

LUMO of $\mathbf{O 7}$

Figure 5: The highest occupied molecular orbitals of molecules in their respective ground states and lowest unoccupied molecular orbitals of molecules in their respective excited states

\section{Transition density matrix}

The transition density matrix provides a spatial map that contains information about chargetransfer lengths $(\Delta r)$ (Table 3 ) and the degree of electron-hole overlap $(\Lambda)$ (Table 4 ) analysis during excitations. The former is the measurement of the separation distance between the electron and its corresponding hole. This chemical index can be used to distinguish between local excitations (LE) and CT. The former occurs if $\Delta r \leq 2 \AA$, and the latter if $\Delta r \geq 2 \AA$ [46]. Table 3 shows the corresponding orbital pairs in which electron-hole separation distance is measured. From Table 3, the degree of electron-hole separation distance lies in the order $04>03>02>05>01>07>06$. The corresponding orbital pairs also agree with the localization and delocalization of $\pi$-electrons for
HOMO and LUMO orbitals in Figure 5. Other than O6, CT is displayed for the orbital pairs in all the compounds. The observation in $\mathbf{O 6}$ does not agree with the fragment-based electron-hole analysis described earlier for this compound. A possible reason could be the level of theory and basis set selected when performing DFT calculations. It is believed that greater separation distance between an electron and its corresponding hole leads to lesser electron-hole overlap, resulting in lower charge recombination [46]. However, Figure 6 clearly shows that such a trend is not observed within our study. When comparing the data for $\Delta r$ to $\Lambda$, only $\mathbf{0 1 , ~} \mathbf{O 2}$ and $\mathbf{O 6}$ followed the expected trend. Previous studies that involve the chemical index $\Lambda$ have shown inaccurate measurements irrespective of the level of theory used [46, 47]. It is also expected that a high single point excitation energy of an electron would induce a large $\Delta r$. Although Figure 7 does not show this trend, only $\mathbf{O 5}$ and $\mathbf{O 7}$ spoil this relationship. Then it is also expected that each compound's anchoring group with the largest concentration of electrons should have the largest $\Delta r$. However, as Figure 8 shows, the selected model failed to verify this relationship, as only $01, \mathbf{O 2}$ and $\mathbf{0 7}$ follow this trend. This significant deviation can be attributed to the direct relationship between $\Lambda$ and electron contributions.

Table 3: Charge-transfer lengths $(\Delta r)$ for the first excited state within each compound

\begin{tabular}{|l|l|l|}
\hline Compound & $\underline{\Delta r(\AA)}$ & $\underline{\text { Orbital pair }}$ \\
\hline O1 & 3.31 & $242 \longrightarrow 251$ \\
\hline O2 & 3.85 & $87 \longrightarrow 88$ \\
\hline O3 & 3.98 & $91 \longrightarrow 92$ \\
\hline O4 & 4.35 & $87 \longrightarrow 88$ \\
\hline O5 & 3.12 & $159 \longrightarrow 165$ \\
\cline { 2 - 3 } & 3.38 & $161 \longrightarrow 165$ \\
\hline O6 & 1.74 & $402 \longrightarrow 404$ \\
\hline
\end{tabular}




\begin{tabular}{|l|l|l|}
\hline O7 & 2.26 & $213 \longrightarrow 217$ \\
\cline { 2 - 3 } & 2.89 & $216 \longrightarrow 217$ \\
\hline
\end{tabular}

Table 4: Degree of electron-hole overlap $(\Lambda)$ for the first excited state within each compound

\begin{tabular}{|l|l|l|}
\hline$\underline{\text { Compound }}$ & $\underline{\Lambda}$ & $\underline{\text { Orbital pair }}$ \\
\hline O1 & 0.324 & $242 \longrightarrow 251$ \\
\hline O2 & 0.506 & $87 \longrightarrow 88$ \\
\hline O3 & 0.471 & $91 \longrightarrow 92$ \\
\hline O4 & 0.280 & $87 \longrightarrow 88$ \\
\hline O5 & 0.150 & $159 \longrightarrow 165$ \\
\cline { 2 - 3 } & 0.118 & $161 \longrightarrow 165$ \\
\hline O6 & 0.0476 & $367 \longrightarrow 404$ \\
\hline \multirow{2}{*}{ 07 } & 0.271 & $213 \longrightarrow 217$ \\
\cline { 2 - 3 } & 0.143 & $216 \longrightarrow 217$ \\
\hline
\end{tabular}

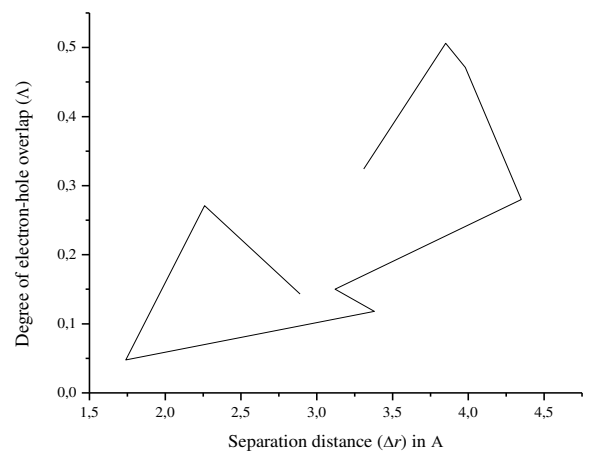

Figure 6: Graphical relationship between electronhole separation distance and degree of electronhole overlap for the orbital pair within each compound

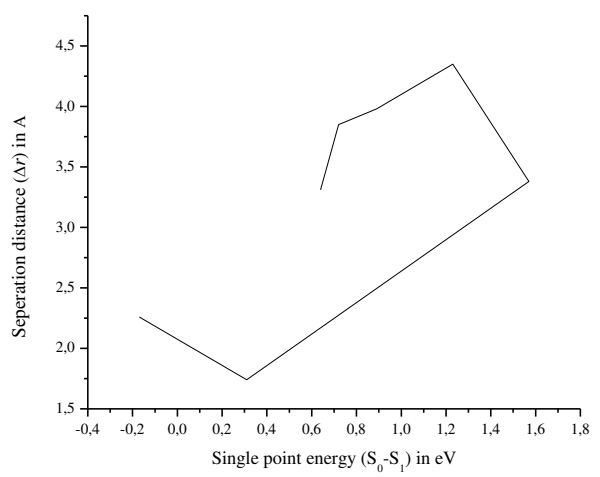

Figure 7: Graphical relationship between single point energy and electron-hole separation distance for the orbital pair within each compound

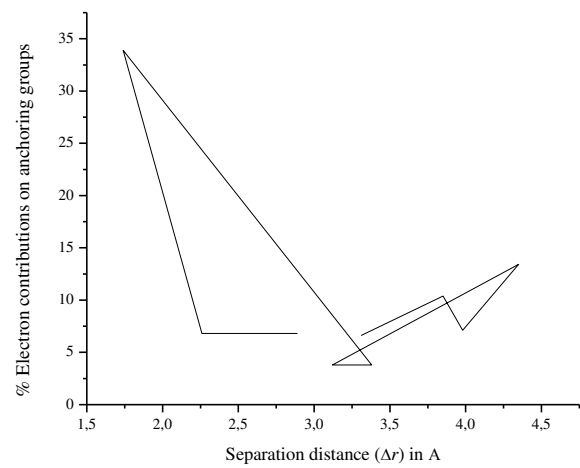

Figure 8: Electron-hole separation distance vs \% electron contribution to anchoring groups within each compound

When acceptor moieties draw electron density from the donor core via the $\pi$-spacer, the transition between different energy states do not change as only the vibrational energy levels change. This allows us to analyze and visualize the dynamics of shifting electron density in terms of $\Delta r$ [41]. Observing the influence of the moiety on the photophysical properties of each molecule will allow us to tune their light-harvesting efficiencies (LHEs) and predict the outcome of charge recombination (i.e. the longer the $\Delta r$, the lower the charge recombination) [48]. A detailed discussion 
on the atom-based transition density matrix is given in the ESI

Figure 9 can be interpreted in the same way as Figure S6. At first glance, the light green colour in the right-hand corner of $\mathbf{O 1}$ seems that the largest electron-hole overlap for the acceptor fragment occurred in this compound, which results in large charge recombination. However, the value in the yaxis indicates that $\mathbf{O 6}$ holds the largest charge recombination in the acceptor fragment. This agrees with the measurement for $\Delta r$, which showed LE. It is also observed that $\mathbf{O 2}$ holds the least charge recombination across the acceptor fragment, although it does not have the largest $\Delta r$ value. This is also verified in Tables S8-S14.

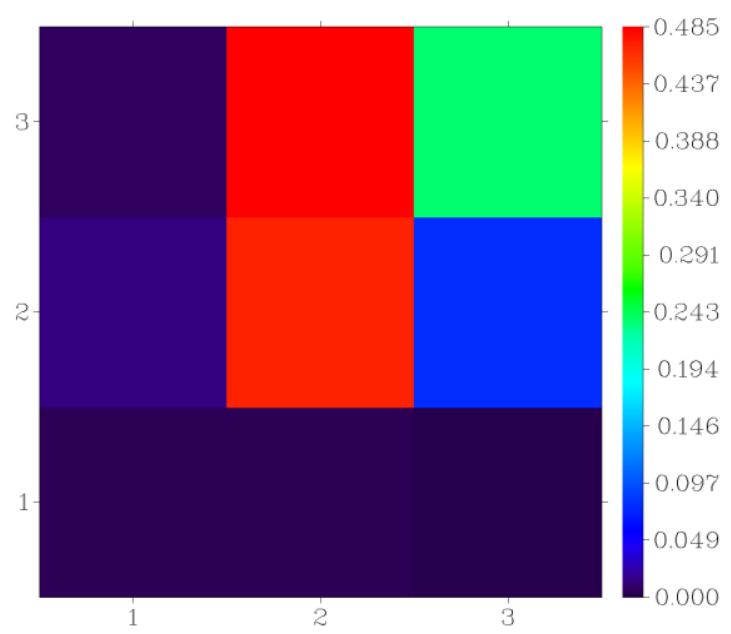

01

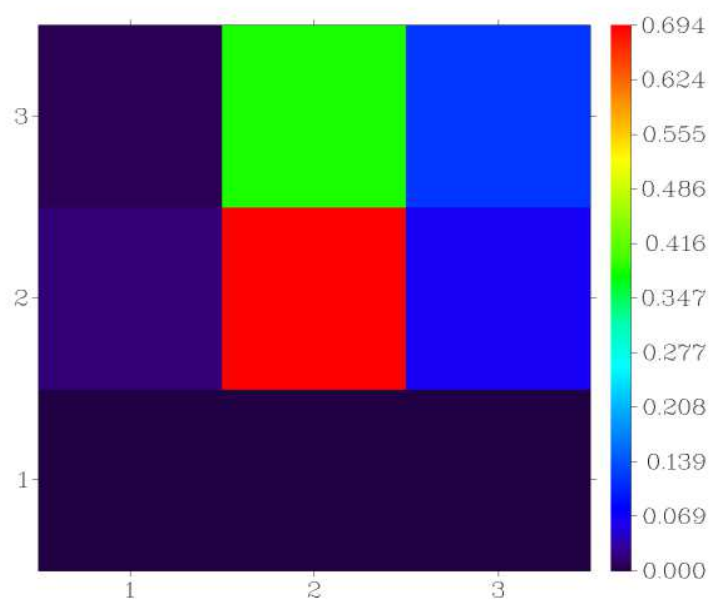

$\mathrm{O2}$

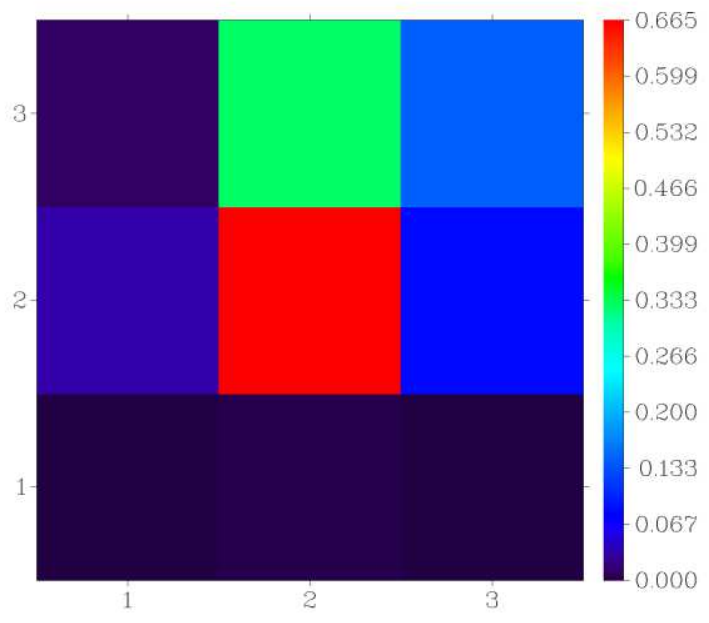

$\mathbf{0 3}$

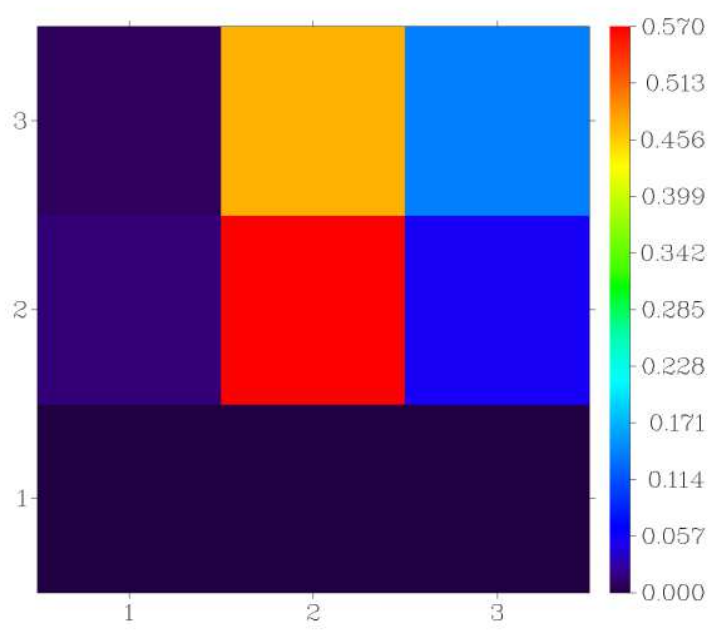

04

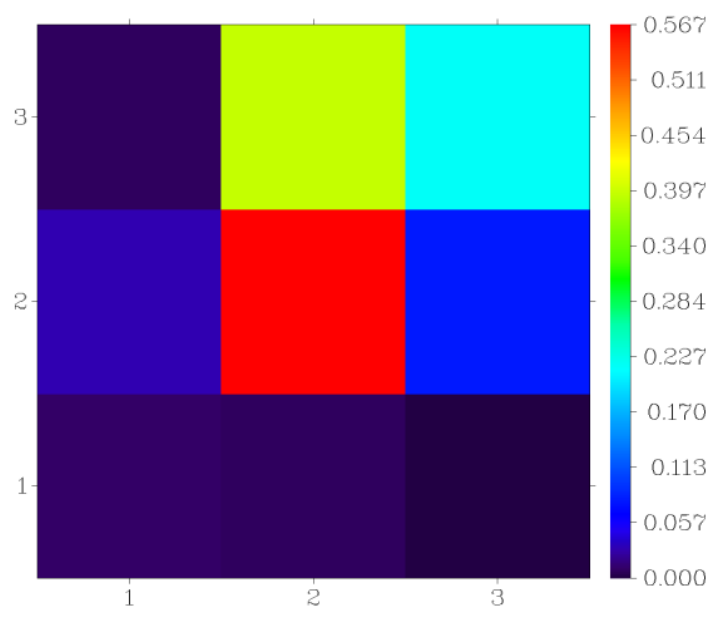

05 


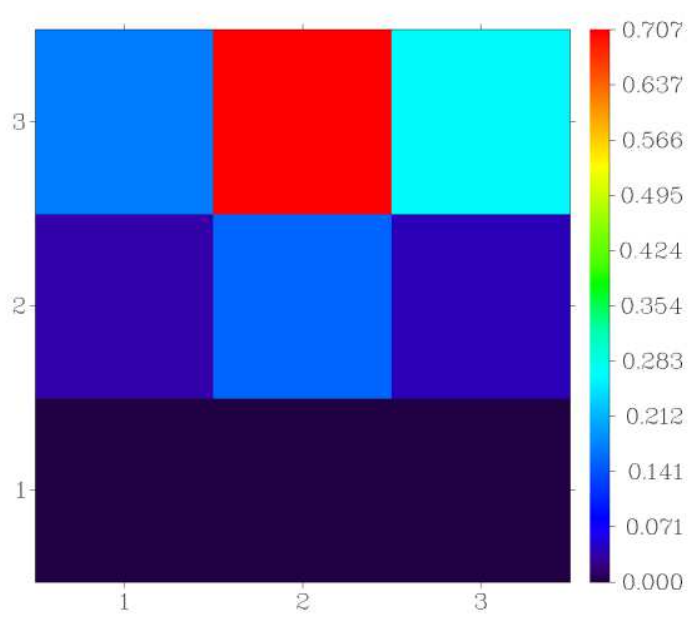

06

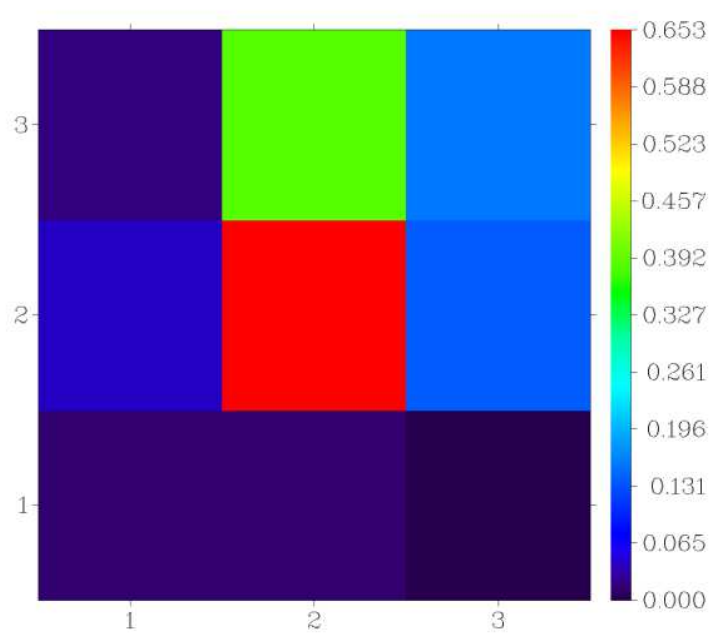

07

Figure 9: Transition density matrix of molecules demonstrating electron distribution from donor core to acceptor moieties

\section{Intra-fragment charge transfer (IFCT) and dihedral angles}

The positive IFCT values in Table 5 indicate a higher concentration of electrons on the acceptor fragment than the donor and $\pi$-spacer fragments. Large IFCT is achieved for smooth electron movements between D and the $\pi$-spacer as well as the $\pi$-spacer and A fragments. Smooth electron movements typically occur for small dihedral angles between $\mathrm{D}-\pi$ and $\pi$-A fragments [22]. This is verified in 04. However, a large IFCT is also observed in 05, although large $D-\pi$ and $\pi$-A dihedral angles are observed. A possible reason for this could be the large concentration of electrons supplied by the arylamine donor group and other factors such as atomic shell structure, local polarizability/hardness, etc. [43]. Although a large IFCT is observed in $\mathbf{0 4}$, it is highly likely that dye aggregation from intermolecular interactions between molecules can occur due to its small D-A dihedral angle, resulting in low electron injection into the conduction band of $\mathrm{TiO}_{2}$ [32]. The largest IFCT value was also observed for O6. This is a bit unexpected due to its small $\Delta r$ value that indicates LE and its large D- $\pi$ and $\pi$-A dihedral angles. Its D-A dihedral angle shows that it is not very prone to intermolecular interactions that lead to charge aggregations. A positive IFCT in $\mathbf{O 7}$ also indicates a possibility for electron injection into the conduction band of $\mathrm{TiO}_{2}$, although large $\mathrm{D}-\pi$ and $\pi$ A dihedral angles were also observed. Furthermore, a reasonable D-A dihedral angle was observed that might be large enough to prevent dye aggregation.

Table 5: Intra-fragment charge transfer and dihedral angles between fragments within compounds

\begin{tabular}{|l|l|l|l|l|}
\hline & $\underline{4}$ & \multicolumn{3}{|l|}{ Dihedral angles $\left(^{\circ}\right)$} \\
\hline$\underline{\text { Compound }}$ & $\underline{(\mathrm{D}-\pi-\mathrm{A})}$ & $\underline{(\mathrm{D}-\pi)}$ & $\underline{(\pi-\mathrm{A})}$ & $\underline{(\mathrm{D}-\mathrm{A})}$ \\
\hline $\mathbf{O 1}$ & 0.35923 & 86.04 & 54.25 & 81.91 \\
\hline $\mathbf{O 2}$ & 0.29634 & 18.06 & 10.02 & 10.93 \\
\hline $\mathbf{O 3}$ & 0.23401 & 89.97 & 0 & 89.97 \\
\hline $\mathbf{O 4}$ & 0.38426 & 14.64 & 14.35 & 8.74 \\
\hline $\mathbf{O 5}$ & 0.27091 & 88.35 & 53.68 & 86.59 \\
\hline $\mathbf{O 6}$ & 0.52267 & 83.11 & 86.07 & 64.25 \\
\hline $\mathbf{O 7}$ & 0.21145 & 65.74 & 40.10 & 37.49 \\
\hline
\end{tabular}

\section{Relationship between charge separation distance and} parameters such as IPCE, charge 
regeneration, $L H E$, electron

\section{injection and charge collection}

We then tested whether there is a correlation between $\triangle r$ and IPCE. It is expected that a large $\Delta r$ will yield a high IPCE value. However, this relationship was spoiled by the outliers 02 and 04 , which caused the graph to display the pattern in Figure 10.

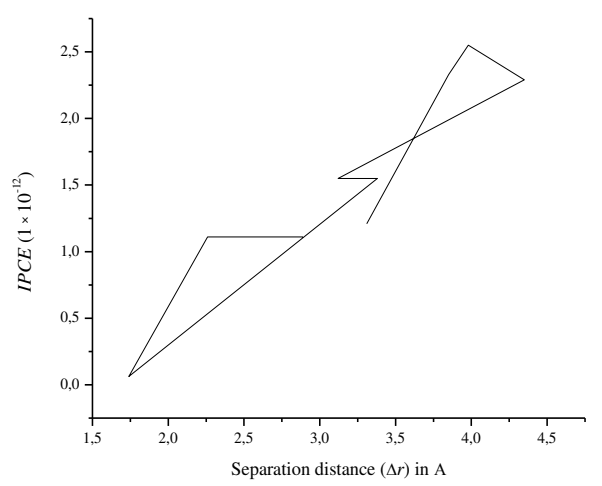

Figure 10: Graphical relationship between electron-hole separation distance and incident photon transfer efficiency

Figure 11: Graphical relationship between electron-hole separation distance and dye regeneration

Since the IPCE comprises LHE, $\Phi_{\text {inj }}$ and $n_{\text {collect }}$, we also tested $\Delta r$ against the latter three parameters. When we tested $\Delta r$ against $L H E$, we observed that $\mathbf{O 3}$ and $\mathbf{O 4}$ were the outliers that caused the pattern that is displayed in Figure 12.

As we expected a large $\Delta r$ to regenerate the dye more easily, we also tested its relationship against $\Delta G_{\text {dye }}^{\text {regen }}$. In this case, $\mathbf{0 3}, \mathbf{O 5}$ and $\mathbf{0 7}$ were the outliers, causing the graph to display the pattern in Figure 11.

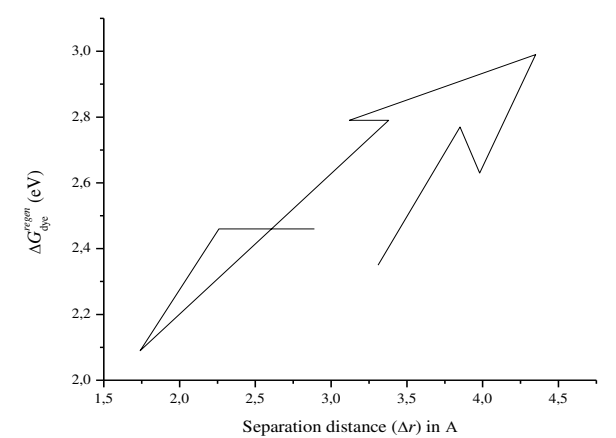

$$
\text { (1) }
$$


Figure 12: Graphical relationship between electron-hole separation distance and lightharvesting efficiency

It is also expected that a larger $\Delta r$ will inject more electrons into the semiconductor's conduction band. The outliers 04 and 07 spoil this relationship, causing the pattern that is displayed in Figure 13.

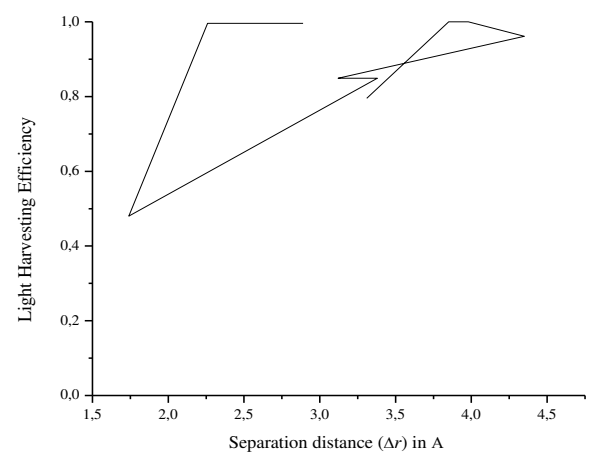

Figure 13: Graphical relationship between electron-hole separation distance and electron injection efficiency

The same correlation between $\Delta r$ and $n_{\text {collect }}$ was expected, but the outliers $\mathbf{O 3}$ and $\mathbf{0 7}$ spoiled this relationship and yielded the graph in Figure 14.

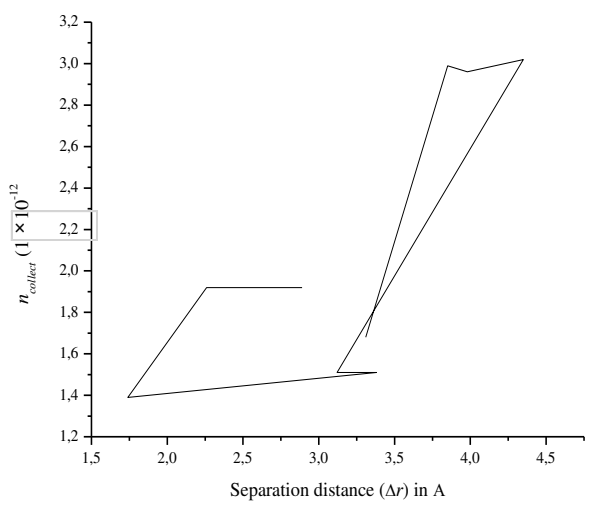

Figure 14: Graphical relationship between electron-hole separation distance and charge collection efficiency

\section{Conclusion} characteristics of compounds that contained 1,3,4oxadiazole moieties. The compounds were constructed and optimized using DFT and TD-DFT calculations. These moieties served as $\pi$-spacer units within each dye of dye-sensitized solar cells (DSSCs). Starburst, Hydroxide, methoxy, amino and triarylamine served as the donor groups, while cyano acrylic acid served as the acceptor group in all the compounds other than $\mathbf{0 7}$, where a cyano group served as the acceptor. The probability of charge recombination was studied by analyzing electron-hole overlaps for specific atoms and fragments. In doing so, the charge-transfer lengths $(\Delta r)$ were used as a chemical index that measures the separation distance between the electron and its corresponding hole. It was assumed that the largest $\Delta r$ will cause the smallest degree of electron-hole overlap $(\Lambda)$, leading to the lowest charge recombination. As $\Lambda$ has shown to be an unreliable chemical index, this correlation was not observed. It was also expected that a large single point excitation energy would cause a large $\Delta r$. Other than a few outliers, this relationship was observed. Then it was assumed that an increase in $\Delta r$ will induce a high electron density around the anchoring groups. Still, due to the relationship between electron contributions and $\Lambda$, this correlation did not hold well. Other than O6, all the compounds have shown $\Delta r$ values above the minimum value for charge transfer (CT) between their fragments, with 04 showing the largest $\Delta r$ value. However, its anchoring groups contained lower electron densities than O6. The large $\Delta r$ value in $\mathbf{O 4}$ also supports its large $\Delta \boldsymbol{G}_{\boldsymbol{d y e}}^{\text {regen }}$ value as the latter play a role in charge recombination. However, the small D-A dihedral angle within this compound might render it inefficient as a dye as intermolecular interactions could occur, leading to aggregation that results in less electrons being injected into the 
semiconductor's conduction band. The bulky sizes of 06 and 07 cause slow diffusion constants $\left(D_{\pi}\right)$ for the movement of $\pi$-electrons, inducing low charge collection efficiencies $\left(n_{\text {collect }}\right)$. However, the anchoring group of $\mathbf{O 6}$ contained the highest concentration of electrons. As IPCE is the key determinant for any compound to be used as a dye, the large ICPE value observed in $\mathbf{O 3}$ indicates that it would serve as the best dye. Since we expected a larger $\Delta r$ to produce a higher IPCE, this was observed in all the compounds except 02 and 04. Similar relationships between $\Delta r$ and the parameters $L H E, \Phi_{\text {inj }}$ and $n_{\text {collect }}$, that comprises the IPCE was also observed in most of the compounds apart from a few outliers. This study serves as a valuable guide to select any compound from a range of compounds to be used as a metal-free organic dye in dye-sensitized solar cells, from which the actual efficiencies can be obtained when studying the DSSC by performing TD-DFT calculations. However, when performing TD-DFT calculations on the compounds that form the DSSC, interactions between the dye, semiconductor and electrolyte will have to be considered.

\section{Acknowledgements}

The authors would like to acknowledge the centre for high-performance computing CHPC, South Africa for providing computational resources for this research project, University of South Africa (UNISA) and the University of Johannesburg (UJ) for their support as well as the national research foundation (NRF) for funding.

\section{Declarations}

Funding acquisition the University of Johannesburg, South Africa.
Conflicts of interests The authors declare no competing interests

Availability of data All data will be available if required

Code Not applicable

Authors' contributions Louis-Charl Coetzee: writing original draft; Adedapo Adeyinka: formal analysis, data curation; Nomampondo Magwa: formal analysis

\section{References}

1. N. Abas, A. Kalair, and N. Khan, Fut., 2015, 69, 31-49

2. N. Robertson, Angew. Chem. Int. Ed., 2006, 45, 2338-2345

3. A.F. Hina, K. Priya, T.B. Sudakar, K.R. Devabalaji, M. Rekha, K. Rajalakshmi, and C. Shilaja, Int. J. Inn. Res. Sci. Eng. Tech., 2014, 3, 1, 1618-1624

4. P.A. Owusu, and S.A. Sarkodie, Cog. Eng., 2016, 3, 1, 1-14

5. Z. Usman, J. Tah, H. Abanda, and C. Nche, Build., 2020, 10, 192, 5, 1-22

6. J.A. Luceno-Sanchez, A.M. Diez-Pascual, and R.P. Capilla, Int. J. Mol. Sci., 2019, 20, 976, 142

7. J.K. Lee, and M. Yang, Mat. Sci. Eng. B., 2011, 176, 1142-1160

8. I.R. Perera, C.V. Hettiarachchi, and R.J.K.U. Ranatunga, Adv. Sol. En. Res., 2018, 175-219

9. S. Ahmad, J. Liu, W. Ji, and L. Sun, Mat., 2018, 11, 1868, 1-8

10. O. Yildirim, M. Bonomo, N. Barbero, C. Atzori, B. Civalleri, F. Bonino, G. Viscardi, and C. Barolo, En., 2020, 13, 5602, 1-48

11. Y. He, Z. Zhang, W. Wang, and L. Fu, J. All. Comp., 2020, 825, 
12. S.K. Sharma, J.E.TI.R., 2017, 4, 11, 1041-1059

13. S.K. Sharma, J.Univ. Shang. Sci. Tech. 2021, 23, 4, 267-277

14. R. Khajavian, M. Mirzaei, and H. Alizadeh, Dalt. Trans., 2020, 49, 13936-13947

15. C.P. lee, C.T. Li, and K.C. Ho, Mat. Tod., 2017, 1-17

16. R. Chauhan, R. Kushwaha, and L. Bahadur, J. En., 2014, 1-10

17. D. Cao, J. Peng, Y. Hong, X. Fang, L. Wang, and H. Meier, Org. Lett., 2011, 13, 1, 16101613

18. S. Manoharan, S. Anandan, Dyes Pigm., 2014, 105, 223-231

19. M. Castillo-Valles, J.M. Andres-Casten, J. Garin, J. Orduna, B. Villacampa, S. Franco, and M.J. Blesa, R.S.C. Adv., 2015, 5, $90667-$ 90670

20. L.L. Tan, J.M. Liu, S.Y. Liu, S.Y. Li, L.M. Xiao, Chem. Sus. Chem., 2014, 8, 2

21. L.L. Tan, J.F. Huang, Y. Shen, L.M. Xiao, J.M. Liu, D.B. Kuang, and C.Y. Su, J. Mat. Chem. A, 2014, 2, 8988-8994

22. N. Kungwan, P. Khongpracha, S. Namuangruk, J. Meeprasert, C. Chitpakalee, and S. Jungsuttiwong, Theor. Chem. Acc., 2014, 133, 1523

23. I.M. Abdellah, A. El-Shafei, J. Photochem. Photobio. A Chem., 2020, 387, 1-10

24. M. Gilbert, and B. Albinsson, Chem. Soc. Rev., 2015, 44, 4, 845-862

25. J. Tang, J. Hua, W. Wu, J. Li, Z. Jin, Y. Lang, and H. Tian, En. Envir. Sci., 2010, 3, 17361745

26. Z. Ning, Q. Zhang, W. Wu, H. Pei, B. Liu, and H. Tian, J. Org. Chem., 2008, 73, 3791-3797

27. P. Naik, M.R. Elmorsy, R. Su, D.D. Babu, A. El-Shafei, A.V. Adhikari, Sol. En., 2017, 153, 600-610

28. P. Naik, R. Su, M.R. Elmorsy, A. El-Shafei, and A.V. Adhikari, Photochem. Photobio. Sci., 2018, 17, 302-314

29. K. Sharma, V. Sharma, and S.S. Sharma, Nan. Res. Lett., 2018, 13, 381, 1-46

30. X. Zarate, S. Schott-Verdugo, A. RodriguesSerrano, and E. Schott, J. Phy. Chem. A, 2016, 120, 1613-1624

31. Y. Koyama, T. Miki, X.F. Wang, and H. Nagae, Int. J. Mol. Sci., 2009, 10, 11, 45754622

32. K. Sanusi, N.O. Fatomi, A.O. Borisade, Y. Tilmaz, U. Ceylan, and A. Fashima, Chem. Phys. Lett., 2019, 1-7

33. T.W. Hamann, A.B.F. Martinson, J.W. Elam, M.J. Pellin, and J.T. Hupp, J. Phys. Chem. C, 2008, 112, 10303-10307

34. U. Mehmood, I.A. Hussein, and M. Daud, Int. J. Photoen., 2015, 1-8

35. K. Srinivas, G. Sivakumar, R.K. Chitumalla, M.A. Reddy, K. Bhanuprakash, J.R. Vaidya, C.W. Chen, Y.C. Hsu, and J.T. Lin, Syn. Met., 2011, 161, 15-16, 1671-1681

36. A. Singh, G. Kociok-Köhn, M. Trivedi, R. Chauhan, A. Kumar, S.W. Gosavi, C. Terashima, and A. Kujishima, N. J. Chem., 2019, 43, 12, 4745-4756

37. L.K. Elizabeth, $\mathrm{PhD}$ thesis, Durham University, 2012

38. M.J. Friesh, G. Truks, H. Schlegel, G. Scuseria, M. Robb, J. Cheeseman, G. Scalmani, V. Barone, B. Mennucci, G. Pettersson: Gaussian 09, Revision D.01, Gaussian Inc.; Wallingford, CT, 2009

39 Lu T, Chen F, J. Theo. Comp. Chem. 2012, 11, 163-183

40 Z. Ning, Q. Zhang, H. Pei, J. Luan, C. Lu, Y. Cui, and H. Tian, J. Phys. Chem. C, 2009, 113, 10307-10313

41 U. Mubashar, A. Farhat, R.A. Khera, N. Iqbal, 
R. Saleem, and J. Iqbal, J. Mol. Mod., 2021, 27, 216, 1-13

42 A. Mathiyalagan, K. Manimaran, K. Muhu, and M. Rajakantham, Res. Chem., 2021, 3, 138

43 F.A. Bulat, J.S. Murray, and P. Politzer, Comp. Theo. Chem., 2021, 1199, 1-6

44 H. Wang, Q. Liu, D. Liu, R. Su, J. Liu, and Y. Li, Int. J. Photoen., 2018, 1-17

45 W. Sharmoukh, J. Cong, B.A. Ali, N.K. Alam, and L. Kloo, Am. Chem. Soc., 2020, 5, 27, 16856-16864

46 C.A. Guido, P. Cortona, B. Mennucci, and C. Adamo, J. Chem. Theo. Comput., 2013, 9,

\section{8-31141}

47 T. Le Bahers, C. Adamo, and I. Ciofini, J. Chem. Theo. Comput., 2011, 7, 2498-2506

48 S. Tretiak, and S. Mukamel, Chem. Rev., 2002, 102, 3171-3212 


\section{Supplementary Files}

This is a list of supplementary files associated with this preprint. Click to download.

- ESIfor134oxadiazoles.docx

- Highlightsof1340xadiazoles.docx 